40th Anniversary Issue

\title{
tmLQCD: A program suite to simulate Wilson twisted mass lattice QCD
}

\author{
Karl Jansen ${ }^{\mathrm{a}}$, Carsten Urbach ${ }^{\mathrm{b}, *}$ \\ a DESY, Patanenallee 6, Zeuthen, Germany \\ ${ }^{\mathrm{b}}$ Humboldt-Universität zu Berlin, Institut für Physik, Newtonstr. 15, 12489 Berlin, Germany
}

\section{A R T I C L E I N F O}

\section{Article history:}

Received 31 March 2009

Received in revised form 19 May 2009

Accepted 20 May 2009

Available online 22 May 2009

PACS:

11.15.-q

11.15. Ha

11.38.-t

11.38.Gc

Keywords:

Hybrid Monte Carlo algorithm

Lattice QCD

\begin{abstract}
A B S T R A C T
We discuss a program suite for simulating Quantum Chromodynamics on a 4-dimensional space-time lattice. The basic Hybrid Monte Carlo algorithm is introduced and a number of algorithmic improvements are explained. We then discuss the implementations of these concepts as well as our parallelisation strategy in the actual simulation code. Finally, we provide a user guide to compile and run the program.
\end{abstract}

\section{Program summary}

Program title: tmLQCD

Catalogue identifier: AEEH_v1_0

Program summary URL:: http://cpc.cs.qub.ac.uk/summaries/AEEH_v1_0.html

Program obtainable from: CPC Program Library, Queen's University, Belfast, N. Ireland

Licensing provisions: GNU General Public Licence (GPL)

No. of lines in distributed program, including test data, etc.: 122768

No. of bytes in distributed program, including test data, etc.: 931042

Distribution format: tar.gz

Programming language: $\mathrm{C}$ and MPI

Computer: any

Operating system: any with a standard C compiler

Has the code been vectorised or parallelised?: Yes. One or optionally any even number of processors may be used. Tested with up to 32768 processors

RAM: no typical values available

Classification: 11.5

External routines: LAPACK [1] and LIME [2] library

Nature of problem: Quantum Chromodynamics

Solution method: Markov Chain Monte Carlo using the Hybrid Monte Carlo algorithm with mass preconditioning and multiple time scales [3]. Iterative solver for large systems of linear equations.

Restrictions: Restricted to an even number of (not necessarily mass degenerate) quark flavours in the Wilson or Wilson twisted mass formulation of lattice QCD.

Running time: Depending on the problem size, the architecture and the input parameters from a few minutes to weeks.

References:

[1] http://www.netlib.org/lapack/.

[2] USQCD, http://usqcd.jlab.org/usqcd-docs/c-lime/.

[3] C. Urbach, K. Jansen, A. Shindler, U. Wenger, Comput. Phys. Commun. 174 (2006) 87, hep-lat/0506011.

(C) 2009 Elsevier B.V. All rights reserved.

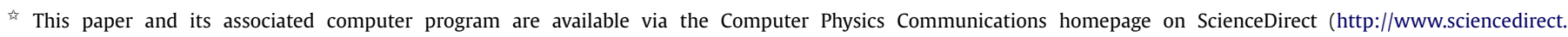
com/science/journal/00104655).

* Corresponding author.

E-mail address: carsten.urbach@physik.hu-berlin.de (C. Urbach).
} 


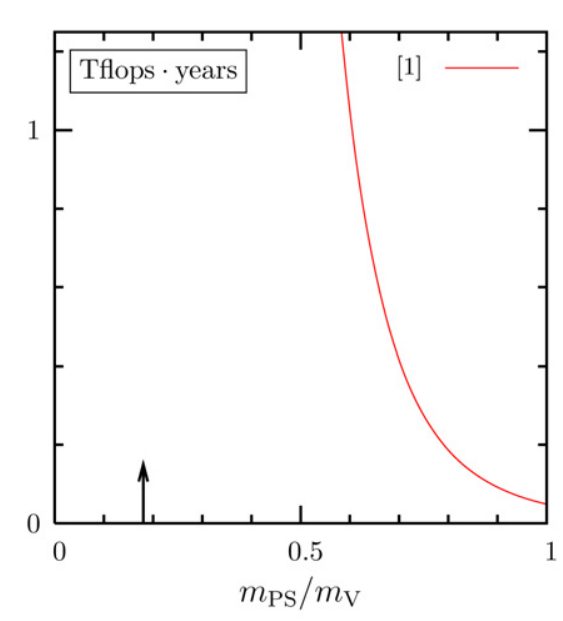

(a)

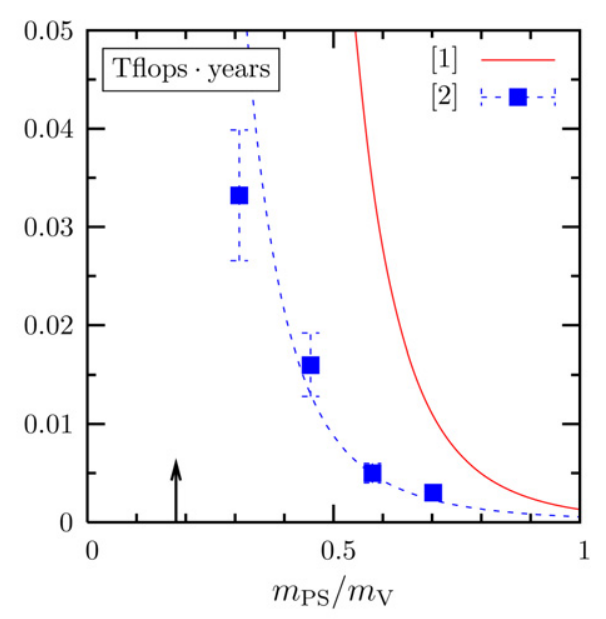

(b)

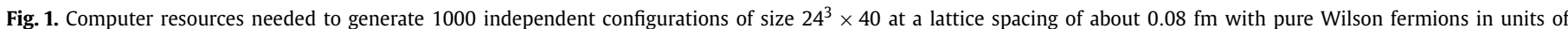

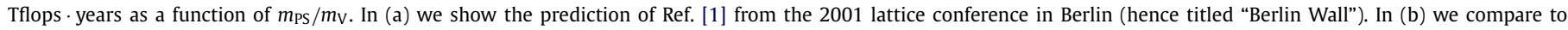

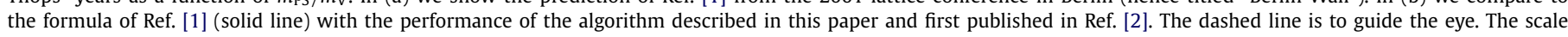

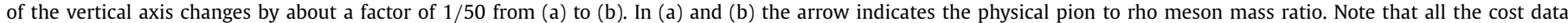
were scaled to match a lattice time extend of $T=40$.

\section{Introduction}

This contribution to the anniversary issue of CPC deals with the strong force in Particle Physics. The strong force is presumably the least well understood fundamental interaction between elementary particles. It is responsible for the existence of protons and neutrons, or more generally all nuclei, as bound states. The constituents of the nuclei are the quarks and gluons as the fundamental particles. It is interesting to observe that the energy (mass) of a proton has a size of about $1 \mathrm{GeV}$ while the mass of the two constituent up and down quarks is at the order of only a few MeV. Hence, the by far biggest contribution to the proton mass is pure binding energy.

This shows already that a description of the proton in terms of the underlying quark and gluon degrees of freedom must be highly non-trivial. The model that is believed to provide a theoretical framework for the strong interaction and should give such a description is Quantum Chromodynamics (QCD). Although this theory can be written in a very compact mathematical form, it is a highly non-linear theory that does not allow for a closed analytical solution.

However, and rather fortunately, QCD can be reformulated in such a way that computational physics methods can be applied to calculate properties of QCD from first principles and without relying on approximations. In this approach, space and time are rendered discrete and a lattice spacing $a$ is introduced. Thus, a 4-dimensional space-time lattice is considered and the quark and gluon degrees of freedom are placed on the lattice points or on so-called links that connect lattice points. In this way one obtains a model of "quark" spins, which are coupled to nearest neighbours only, very much reminiscent of an Ising model in statistical physics. Indeed, the methods of statistical physics, namely the evaluation of the partition function by means of numerical simulations using Monte Carlo methods employing importance sampling, are the key to address QCD on the 4-dimensional lattice, which we shall refer to as lattice QCD (LQCD).

Although the concepts to treat LQCD numerically are very clear, the problem has an intrinsically extremely high computational demand. The crucial factor is that in the end the introduced discretisation has to be removed again. If we consider a lattice with say, $L=3$ fm linear extent and a lattice spacing of $a=0.1 \mathrm{fm}$ then we would have to use $N=3 / 0.1=30$ lattice points in one direction. Since we are dealing with a 4-dimensional problem, we need hence $30^{4}$ lattice points for such a more or less reasonable physical situation. Simulations on such a lattice require in LQCD already several Teraflops. Keeping $L=3 \mathrm{fm}$ fixed and decreasing the lattice spacing to remove the discretisation by, say, a factor of two increases the cost of the simulation already by a factor $2^{4}$. As this would not be worse enough, the used algorithms contribute another factor of $2^{(2-3)}$. Hence, going to really fine discretisations where the effects of the non-zero lattice spacing can safely be neglected or at least a controlled extrapolation to zero values of the lattice spacing can be performed is an extremely demanding computational challenge which will finally require at least Petaflops computing, an area of computing power we are realising today.

However, even with the advent of Petaflops computers, the goal of "solving" QCD on a lattice would be completely out of reach without some algorithmic improvements that were invented in recent years. This is shown in Fig. 1. In the left panel of the graph, the number of Teraflops years for a certain typical simulation is shown as a function of the ratio of two meson masses, the pseudo scalar and the vector meson. The graph derives from the known cost of the used algorithm in the year 2001 [1].

It is important to realise that the meson mass ratio used in Fig. 1 assumes a value of about 0.2 in nature as measured in experiments, which is indicated by the arrow in both panels of Fig. 1. The figure clearly demonstrates the strongly growing cost of the simulations when the real physical situation is to be reached. In fact, simulations directly at the physical value of this mass ratio were impossible in 2001 . The right panel of the graph demonstrates the change of the situation when algorithmic improvements are used as the ones described in this article. In fact, the simulation costs shown in the right panel of Fig. 1 originate from direct performance measurements of the code that is described here. As the figure demonstrates, although the simulations at the physical value of the meson mass ratio are still rather demanding, they become clearly realistic with todays Petaflops systems. There are other approaches to improve the HMC algorithm with similar results [3-8]. Very promising is the recent additional improvement using inexact deflation presented in Ref. [9]. 
The algorithmic improvements provided therefore a tremendous gain opening a way for simulations in LQCD that were unthinkable a few years ago. It is precisely the goal of this contribution to describe one programme version of the underlying Hybrid Monte Carlo (HMC) algorithm where a number of such improvements have been incorporated and to make the corresponding code publicly available.

\section{Theoretical background}

\subsection{QCD on a lattice}

Quantum Chromodynamics on a hyper-cubic Euclidean space-time lattice of size $L^{3} \times T$ with lattice spacing $a$ is formally described by the action

$$
S=S_{\mathrm{G}}[U]+a^{4} \sum_{x} \bar{\psi} D[U] \psi
$$

with $S_{\mathrm{G}}$ some suitable discretisation of the Yang-Mills action $F_{\mu \nu}^{2} / 4$ [10]. The particular implementation we are using can be found below in Section 4.2 and consists of plaquette and rectangular shaped Wilson loops with particular coefficients. $D$ is a discretisation of the Dirac operator, for which Wilson originally proposed [11] to use the so called Wilson Dirac operator

$$
D_{W}[U]=\frac{1}{2}\left[\gamma_{\mu}\left(\nabla_{\mu}+\nabla_{\mu}^{*}\right)-a \nabla_{\mu}^{*} \nabla_{\mu}\right]
$$

with $\nabla_{\mu}$ and $\nabla_{\mu}^{*}$ the forward and backward gauge covariant difference operators, respectively:

$$
\begin{aligned}
& \nabla_{\mu} \psi(x)=\frac{1}{a}[U(x, x+a \hat{\mu}) \psi(x+a \hat{\mu})-\psi(x)], \\
& \nabla_{\mu}^{*} \psi(x)=\frac{1}{a}\left[\psi(x)-U^{\dagger}(x, x-a \hat{\mu}) \psi(x-a \hat{\mu})\right],
\end{aligned}
$$

where we denote the SU(3) link variables by $U_{x, \mu}$. We shall set $a \equiv 1$ in the following for convenience. Discretising the theory is by far not a unique procedure. Instead of Wilson's original formulation one may equally well chose the Wilson twisted mass formulation and the corresponding Dirac operator [12]

$$
D_{\mathrm{tm}}=\left(D_{W}[U]+m_{0}\right) 1_{f}+i \mu_{q} \gamma_{5} \tau^{3}
$$

for a mass degenerate doublet of quarks. We denote by $m_{0}$ the bare (Wilson) quark mass, $\mu_{q}$ is the bare twisted mass parameter, $\tau^{i}$ the $i$ th Pauli matrix and $1_{f}$ the unit matrix acting in flavour space (see Appendix A for our convention). In the framework of Wilson twisted mass QCD only flavour doublets of quarks can be simulated, however, the two quarks do not need to be degenerate in mass. The corresponding mass non-degenerate flavour doublet reads [13]

$$
D_{h}(\bar{\mu}, \bar{\epsilon})=D_{\mathrm{W}} 1_{f}+i \bar{\mu} \gamma_{5} \tau^{3}+\bar{\epsilon} \tau^{1}
$$

Note that this notation is not unique. Equivalently - as used in Ref. [14] - one may write

$$
D_{h}^{\prime}\left(\mu_{\sigma}, \mu_{\delta}\right)=D_{\mathrm{W}} \cdot 1_{f}+i \gamma_{5} \mu_{\sigma} \tau^{1}+\mu_{\delta} \tau^{3},
$$

which is related to $D_{h}$ by $D_{h}^{\prime}=\left(1+i \tau^{2}\right) D_{h}\left(1-i \tau^{2}\right) / 2$ and $\left(\mu_{\sigma}, \mu_{\delta}\right) \rightarrow(\bar{\mu},-\bar{\epsilon})$.

\subsection{The Hybrid Monte Carlo algorithm}

For the purpose of introducing the Hybrid Monte Carlo (HMC) algorithm we shall consider only the Wilson twisted mass formulation of lattice QCD with one doublet of mass degenerate quarks with bare quark mass $m_{0}$ and bare twisted mass $\mu_{q}$. The extension to more than one flavour doublet of quarks is straightforward. The corresponding polynomial HMC algorithm used for simulating the mass nondegenerate flavour doublet is discussed in the following sub-section.

After integrating out the Grassmann valued fermion fields, in lattice QCD one needs to evaluate the integral

$$
\int \mathcal{D} U \operatorname{det}\left(Q^{\dagger} Q\right) e^{-S_{G}}
$$

by Markov Chain Monte Carlo methods with some discretisation of the Yang-Mills gauge action $S_{G}$ and

$$
Q \equiv \gamma_{5} D_{\mathrm{W}}[U]+\gamma_{5} m_{0}+i \mu_{q}
$$

with the Wilson-Dirac operator $D_{\mathrm{W}}$ of Eq. (2). Note that $Q$ acts now on one flavour only. The determinant can be re-expressed using complex valued, so-called pseudo fermion fields $\phi$ and $\phi^{\dagger}$

$$
\operatorname{det}\left(Q^{2}\right) \propto \int \mathcal{D} \phi \mathcal{D} \phi^{\dagger} e^{-\left(Q^{-1} \phi, Q^{-1} \phi\right)}
$$

where $S_{\mathrm{PF}} \equiv\left|Q^{-1} \phi\right|^{2}$ is called the pseudo fermion action. The HMC algorithm [15] is then defined by introducing traceless hermitian momenta $P_{x, \mu}$ (conjugate to the fundamental fields $U_{x, \mu}$ ) and a Hamiltonian

$$
\mathcal{H}(U, P)=\sum_{x, \mu} \frac{1}{2} \operatorname{Tr}\left[P_{x, \mu}^{2}\right]+S_{\mathrm{G}}[U]+S_{\mathrm{PF}}[U] .
$$


Given $\mathcal{H}$, the algorithm is composed out of a molecular dynamics update of the fields $(U, P) \rightarrow\left(U^{\prime}, P^{\prime}\right)$ and a Metropolis accept/reject step with respect to $\mathcal{H}$ using the acceptance probability

$$
P_{\text {acc }}=\min \left(1, \exp \left(\mathcal{H}\left(U^{\prime}, P^{\prime}\right)-\mathcal{H}(U, P)\right)\right) \text {. }
$$

While the momenta $P$ are generated at the beginning of a trajectory - in the so called heat-bath step - randomly from a Gaussian distribution, the pseudo fermion fields $\phi$ are generated by first generating random fields $r$ and then

$$
\phi=Q r
$$

such that $\exp \left\{-\left(Q^{-1} \phi, Q^{-1} \phi\right)\right\}=\exp \left\{r^{\dagger} r\right\}$. Note that the pseudo fermion fields are not evolved during the molecular dynamics part of the HMC algorithm.

\subsubsection{Molecular dynamics update}

In the molecular dynamics (MD) part of the HMC algorithm the momenta and gauge fields are updated corresponding to the Hamiltonian equations of motion

$$
\begin{aligned}
\frac{d}{d \tau} P_{x, \mu} & =-F(x, \mu), \\
\frac{d}{d \tau} U_{x, \mu} & =P_{x, \mu} U_{x, \mu}
\end{aligned}
$$

with respect to a fictitious computer time $\tau$ and forces $F$, which are obtained by differentiating the action with respect to the gauge fields $U$, and take values in the Lie algebra of SU(3). The differentiation $D_{U}$ of some function $f(U)$ is defined as

$$
D_{U}^{a} f(U)=\left.\frac{\partial}{\partial \alpha} f\left(e^{i \alpha t^{a}} U\right)\right|_{\alpha=0}
$$

where $t^{a}$ are the generators of $\mathrm{su}(3)$.

Since these equations can in general not be integrated analytically, one uses numerical integration methods, which must be area preserving and reversible. Symmetrised symplectic integrators fulfil these requirements with the simplest example being the leap-frog algorithm. The basic discrete update steps with integration step size $\Delta \tau$ of the gauge field and the momenta can be defined as

$$
\begin{aligned}
& T_{\mathrm{U}}(\Delta \tau): U \quad \rightarrow \quad U^{\prime}=\exp (i \Delta \tau P) U, \\
& T_{\mathrm{S}}(\Delta \tau): P \quad \rightarrow \quad P^{\prime}=P-i \Delta \tau F .
\end{aligned}
$$

The leap-frog algorithm is then obtained by sequential application of

$$
T=T_{\mathrm{S}}(\Delta \tau / 2) T_{\mathrm{U}}(\Delta \tau) T_{\mathrm{S}}(\Delta \tau / 2),
$$

i.e. for a trajectory of length $\tau$ one needs to apply $T^{N_{\mathrm{MD}}}$ with $N_{\mathrm{MD}}=\tau / \Delta \tau$.

\subsubsection{Preconditioning and multiple time scales}

Preconditioning is usually performed by factorising

$$
\operatorname{det}\left(Q^{\dagger} Q\right)=\operatorname{det}\left(R_{1}^{\dagger} R_{1}\right) \cdot \operatorname{det}\left(R_{2}^{\dagger} R_{2}\right) \cdots \operatorname{det}\left(R_{n}^{\dagger} R_{n}\right)
$$

with suitably chosen $R_{1}, R_{2}, \ldots, R_{n}$. Then, for every $R_{i}$ a separate pseudo fermion field $\phi_{i}$ is introduced, such that the Hamiltonian reads

$$
\mathcal{H}(U, P)=\sum_{x, \mu} \frac{1}{2} \operatorname{Tr}\left[P_{x, \mu}^{2}\right]+S_{\mathrm{G}}[U]+\sum_{i=1}^{n} S_{\mathrm{PF}_{i}}
$$

and the equations of motion are changed to

$$
\begin{aligned}
\frac{d}{d \tau} P_{x, \mu} & =-\sum_{i=0}^{n} F_{i}(x, \mu), \\
\frac{d}{d \tau} U_{x, \mu} & =P_{x, \mu} U_{x, \mu},
\end{aligned}
$$

where we identify $F_{0}$ with the force stemming from the gauge action $S_{\mathrm{G}}$.

The factorisation in Eq. (14) can be achieved in many different ways, see for instance Refs. [3-8]. Here we shall only discuss what is known as mass preconditioning or Hasenbusch trick [16-18]. It is obtained by writing the identity

$$
\operatorname{det}\left(Q^{\dagger} Q\right)=\operatorname{det}\left(W^{\dagger} W\right) \cdot \frac{\operatorname{det}\left(Q^{\dagger} Q\right)}{\operatorname{det}\left(W^{\dagger} W\right)}
$$

where

$$
W=D_{\mathrm{W}}+m_{0}+i \mu_{2} \gamma_{5}, \quad \mu_{2}>\mu_{q} .
$$

By adjusting the additional mass parameter $\mu_{2}$, the condition number of $W^{\dagger} W$ and $\left(Q^{\dagger} Q\right) /\left(W^{\dagger} W\right)$ can both be reduced with respect to the one of $Q^{\dagger} Q$ alone. As argued in Ref. [19], the optimal choice leads to a condition number of $\sqrt{k}$ for both $W^{\dagger} W$ and $\left(Q^{\dagger} Q\right) /\left(W^{\dagger} W\right)$, 
where $k$ is the condition number of $Q^{\dagger} Q$. A reduced condition number leads to reduced force contributions in the MD evolution and allows hence for larger values of $\Delta \tau$.

It is important to notice that evaluating the force contribution stemming from $\left(Q^{\dagger} Q\right) /\left(W^{\dagger} W\right)$ is more expensive in terms of computer time than the evaluation of the contribution from $W^{\dagger} W$, since it involves the iterative solution of $\varphi=\left(Q^{\dagger} Q\right)^{-1} \phi$ with the large condition number $k$. Thus, the algorithm might be further improved by not tuning the condition numbers equal as explained beforehand, but by introducing a multiple time scale integration scheme as follows.

Considering a Hamiltonian like in Eq. (15) we may introduce $n+1$ timescales $\Delta \tau_{i}$ with

$$
\Delta \tau_{i}=\frac{\tau}{N_{\mathrm{MD}_{i}}}, \quad N_{\mathrm{MD}_{i}}=N_{n} \cdot N_{n-1} \cdots N_{i}
$$

and basic discrete update steps

$$
\begin{aligned}
& T_{\mathrm{U}}(\Delta \tau): U \quad \rightarrow \quad U^{\prime}=\exp (i \Delta \tau P) U \\
& T_{\mathrm{S}_{i}}(\Delta \tau): P \quad \rightarrow \quad P^{\prime}=P-i \Delta \tau F_{i}
\end{aligned}
$$

with $0 \leqslant i \leqslant n$. We have identified $S_{0} \equiv S_{\mathrm{G}}$. The leap frog update on timescale $i$ is then recursively defined as

$$
T_{i}= \begin{cases}T_{\mathrm{S}_{i}}\left(\Delta \tau_{i} / 2\right) T_{\mathrm{U}}\left(\Delta \tau_{i}\right) T_{\mathrm{S}_{i}}\left(\Delta \tau_{i} / 2\right) & i=0 \\ T_{\mathrm{S}_{i}}\left(\Delta \tau_{i} / 2\right)\left[T_{i-1}\right]^{N_{i-1}} T_{\mathrm{S}_{i}}\left(\Delta \tau_{i} / 2\right) & 0<i \leqslant n\end{cases}
$$

and the full trajectory of length $\tau$ is eventually achieved by $\left[T_{n}\right]^{N_{n}}$.

As was shown in Ref. [2] - and for other factorisations of the determinant in Refs. $[3,7,20]$ - the combination of multiple time scale integration and a determinant factorisation allows to set the algorithm up such that the most expensive operator contributes least to the MD forces. It can then be integrated on the outermost timescale and must be less often inverted.

\subsubsection{Integration schemes}

During the last paragraphs we have introduced the simplest reversible and area preserving integration scheme, known as leap frog integration scheme. There are more involved integration schemes available, partly or completely cancelling higher order discretisation errors.

It turns out that completely cancelling higher order effects is not necessary and often even not efficient. Integration schemes with reduced errors are for example the so called $n$th order minimal norm integration schemes, for details see Ref. [21] and references therein. The second order minimal norm ( $2 \mathrm{MN})$ integration scheme is based on the update step

$$
\begin{aligned}
& T_{0}^{2 \mathrm{MN}}=T_{\mathrm{S}_{0}}\left(\lambda_{0} \Delta \tau_{0}\right) T_{\mathrm{U}}\left(\Delta \tau_{0} / 2\right) T_{\mathrm{S}_{0}}\left(\left(1-2 \lambda_{0}\right) \Delta \tau_{0}\right) T_{\mathrm{U}}\left(\Delta \tau_{0} / 2\right) T_{\mathrm{S}_{0}}\left(\lambda_{0} \Delta \tau_{0}\right), \\
& T_{i}^{2 \mathrm{MN}}=T_{\mathrm{S}_{i}}\left(\lambda_{i} \Delta \tau_{i}\right)\left[T_{i-1}^{2 \mathrm{MN}}\right]^{N_{i-1}} T_{\mathrm{S}_{i}}\left(\left(1-2 \lambda_{i}\right) \Delta \tau_{i}\right)\left[T_{i-1}^{2 \mathrm{MN}}\right]^{N_{i-1}} T_{\mathrm{S}_{i}}\left(\lambda_{i} \Delta \tau_{i}\right),
\end{aligned}
$$

$\lambda_{i}$ is a dimensionless parameter and the $2 \mathrm{MN}$ scheme coincides with the Sexton-Weingarten scheme [22] in case $\lambda_{i}=1 / 6$. The optimal value for $\lambda_{i}$ was given in Ref. [21] to be around 0.19. But its value is likely to depend on the mass values and the time scale under consideration. Note that there is one parameter $\lambda_{i}$ for each timescale $\Delta \tau_{i}$, which can be tuned separately.

While all the integration schemes introduced so far were based on the order $T_{S} T_{U} T_{S}$, it is also possible to revert this order. In this case one talks about the position version of the corresponding integration scheme, while the usual one is called the velocity version. Under certain circumstances they can be more efficient, because one less application of $T_{S}$ is needed. The corresponding update steps can be easily derived from the formulae provided above.

\subsection{Polynomial HMC for a non-degenerate doublet}

In the framework of Wilson twisted mass fermions it is only possible to simulate flavour doublets of quarks. Hence, if one wants to include the strange quark in the simulation one also needs to include the charm. The corresponding mass non-degenerate doublet was defined in Eq. (5). Simulating such a flavour doublet operator is possible using the polynomial HMC (PHMC) algorithm [23-25]. The basic problem that occurs in the mass non-degenerate case is that a single flavour has to be taken into account or equivalently the determinant of a single operator $Q$ needs to be treated. The PHMC algorithm can solve this problem elegantly.

The idea of the PHMC is based on writing

$$
\operatorname{det}(Q)=\operatorname{det}\left(\sqrt{Q^{2}}\right) \approx \operatorname{det}\left(P_{\epsilon, n}^{-1}\left(Q^{2}\right)\right) \propto \int \mathcal{D} \phi \mathcal{D} \phi^{\dagger} e^{-\phi^{\dagger} P \phi},
$$

valid as long as $Q$ is positive. $P_{\epsilon, n}\left(Q^{2}\right)$ is a polynomial approximation of $1 / \sqrt{Q^{2}}$ of degree $n$ in the interval $[\epsilon, 1]$

$$
P_{n, \epsilon}(s)=\frac{1}{\sqrt{s}}\left\{1+R_{n, \epsilon}\right\}, \quad s=Q^{2} .
$$

$R_{n, \epsilon}$ is the error term. It can be shown that for the case of Chebysheff polynomials $|R|$ vanishes exponentially fast with the degree $n$ (for large $n$ ). For more details regarding this issue we refer the reader for instance to Refs. [26,27] and references therein.

It is worth noticing that representing an inverse operator by a polynomial has conceptual advantages. It allows to treat certain regions of the eigenvalue spectrum of the operator in different ways and to separate therefore the infrared from the bulk and ultraviolet parts of the spectrum. Although this has been the main underlying idea of the PHMC algorithm [23-25] we will use it here, however, only as a technical tool to treat single flavours in the simulations. 
For our purpose - introducing $Q_{h}=\gamma_{5} D_{h}$ - we can rewrite the corresponding determinant

$$
\operatorname{det}\left(Q_{h}\right) \propto \int \mathcal{D} \Phi^{\dagger} \mathcal{D} \Phi e^{-\Phi^{\dagger} P_{n, \epsilon}(s) \Phi},
$$

with $s=Q_{h}^{\dagger} Q_{h}$ and the pseudo fermion fields $\Phi$ are now two flavour fields. Note that $D_{h}^{\dagger}=\tau^{1} \gamma_{5} D_{h} \gamma_{5} \tau^{1}$. The application of the polynomial $P$ to a pseudo fermion field $\Phi$ can be performed by either using the Clenshaw recursion relation [28], or by using the product representation

$$
P_{n, \epsilon}(s) \Phi=\left[\prod_{i=1}^{n} c\left(s-z_{i}\right)\right] \Phi \equiv B(s) \cdot B(s)^{\dagger} \Phi
$$

with $z_{i}$ the complex roots of $P$ and a suitably chosen normalisation constant $c$. The product representation is conveniently used in the MD update. For the choice of polynomials, the determination of their roots and how to order them to avoid round-off errors see Appendix C.

The HMC algorithm requires an area preserving and reversible MD update procedure, however, there is no need to use in the MD update the same operator as in the heat-bath step. As long as the acceptance rate is sufficiently high, we are free to use any other operator in the update. In order to exploit this possibility we introduce a second more precise polynomial

$$
\tilde{P}_{m, \delta}(s)=\frac{1}{P_{n, \epsilon} \sqrt{s}}\left\{1+\tilde{R}_{m, \delta}\right\}
$$

which is used in the heat-bath step to generate the pseudo fermion fields from a random field $R$

$$
\Phi=\tilde{P} B^{\dagger} Q_{h} R
$$

and in the acceptance step. The less precise polynomial $P$ is then used only in the MD update.

The polynomial degrees $n, m$ and the approximation intervals have to be determined such as to guarantee a good approximation of $1 / \sqrt{s}$ in the range of eigenvalues of $Q_{h}^{\dagger} Q_{h}$. One may also adopt a strategy to chose $\epsilon$ or $\delta$ larger than a few lowest eigenvalues of $Q_{h}^{\dagger} Q_{h}$ and use re-weighting to correct for this $[23,24]$.

\subsubsection{Even/odd preconditioning}

The (P)HMC algorithm is implemented using even/odd preconditioning [29,30], which is discussed shortly in Appendix B. We want to stress that although even/odd preconditioning is a rather technical step, it leads to a very important improvement of the algorithm performance and is a cornerstone of all HMC implementations in the field.

\subsection{Boundary conditions}

The theory is discretised and put on a finite, hyper-cubic space-time lattice with extensions $L^{3} \times T \equiv \prod_{\mu} L_{\mu}$. The boundary conditions for the gauge fields $U_{x, \mu}$ are chosen to be periodic, i.e.

$$
U_{x+L_{v} \hat{v}, \mu}=U_{x, \mu},
$$

where $\hat{v}$ is a unit vector in direction $\nu$. For the fermionic fields $\psi(x)$ we allow for more general boundary conditions, namely so called twisted boundary conditions

$$
\psi\left(x+L_{\nu} \hat{v}\right)=e^{i \theta_{v} \pi} \psi(x) .
$$

Periodic boundary conditions correspond to $\theta_{v}=0$, while anti-periodic boundary conditions are achieved by setting $\theta_{v}=1$. More generally one can realise with twisted boundary conditions arbitrary values of momentum transfer on the lattice by a convenient re-interpretation of the phases [31].

\section{Overview of the software structure}

The general strategy of the tmLQCD package is to provide programs for the main applications used in lattice QCD with Wilson twisted mass fermions. The code and the algorithms are designed to be general enough such as to compile and run efficiently on any modern computer architecture. This is achieved code-wise by using standard C as programming language and for parallelisation the message passing interface (MPI) standard version 1.1.

Performance improvements are achieved by providing dedicated code for certain widely used architectures, like PC's or the Blue Gene family. Dedicated code is mainly available for the kernel routine - the application of the Dirac operator, which will be discussed in detail in Section 4.1, and for the communication routines.

The tmLQCD package provides three main applications. The first is an implementation of the (P)HMC algorithm, the second and the third are executables to invert the Wilson twisted mass Dirac operator (4) and the non-degenerate Wilson twisted mass Dirac operator (5), respectively. All three do have a wide range of run-time options, which can be influenced using an input file. The syntax of the input file is explained in the documentation which ships with the source code. The relevant input parameters will be mentioned in the following where appropriate, to ease usage.

We shall firstly discuss the general layout of the three aforementioned applications, followed by a general discussion of the parallelisation strategy used in all three of them. 
Read Input From File

Initialise Programme / Read Data from Disk

Do No. of Trajectories times

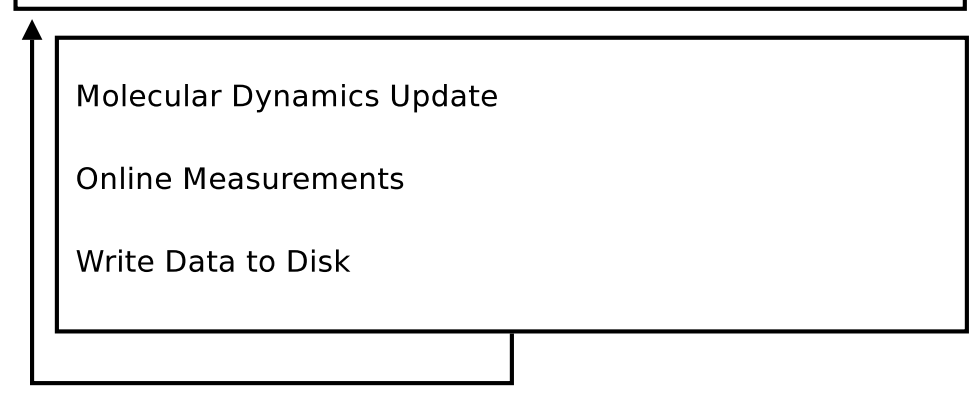

Finalise

Fig. 2. Flowchart for the hmc_tm executable.

\section{1. $h m c_{-} t m$}

In Fig. 2 the programme flow of the hmc_tm executable is depicted. In the first block the input file is parsed and parameters are set accordingly. Then the required memory is allocated and, depending on the input parameters, data is read from disk in order to continue a previous run.

The main part of this application is the molecular dynamics update. For a number of trajectories, which must be specified in the input file, first a heat-bath is performed, then the integration according to the equations of motion using the integrator as specified in the input file, and finally the acceptance step.

After each trajectory certain online measurements are performed, such as measuring the plaquette value. Other online measurements are optional, like measuring the pseudo scalar correlation function.

\subsubsection{Command line arguments}

The programme offers command line options as follows:

- $-\mathrm{h} \mid$ ? prints a help message and exits.

- $-\mathrm{f}$ input file name. The default is hmc. input.

- - the prefix of the output filenames. The default is output. The code will generate or append to two files, output. data and output.para.

\subsubsection{Input/output}

The parameters of each run are read from an input file with default name hmc.input. If it is missing all parameters will be set to their default values. Any parameter not set in the input file will also be set to its default value.

During the run the hmc_tm program will generate two output files, one called per default output. data, the other one output. para. Into the latter important parameters will be written at the beginning of the run.

The file output. data has several columns with the following meanings

(1) Plaquette value.

(2) $\Delta H$.

(3) $\exp (-\Delta H)$.

(4) A pair of integers for each pseudo fermion monomial. The first integer of each pair is the sum of solver iterations needed in the acceptance and heat-bath steps, the second is the sum of iterations needed for the force computation of the whole trajectory.

(5) Acceptance (0 or 1 ).

(6) Time in seconds needed for this trajectory.

(7) Value of the rectangle part in the gauge action, if used.

Every new run will append its numbers to an already existing file.

In addition, the program will create a file history_hmc_tm. This file provides a mapping between the configuration number and its plaquette and Polyakov loop values. Moreover the simulation parameters are stored there and in case of a reread the time point can be found there.

After every trajectory the program will save the current configuration in the file conf . save. 
Do No. of Measurements times

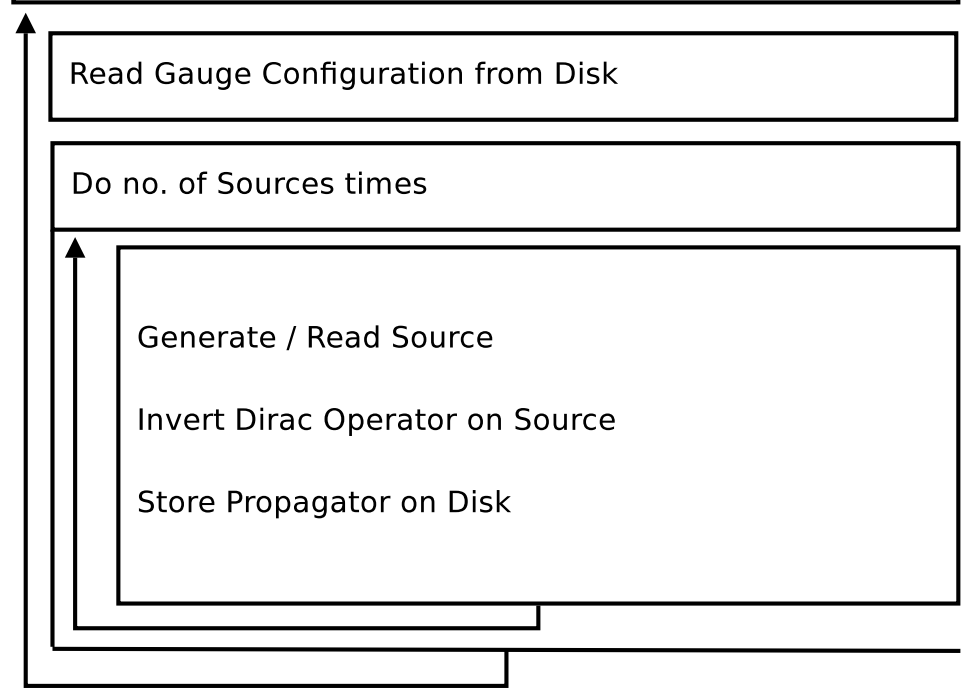

Fig. 3. Flowchart for the main part of the invert and invert_doublet executables.

\section{2. invert and invert_doublet}

The two applications invert and invert_doublet are very similar. The main difference is that in invert the one flavour Wilson twisted mass Dirac operator is inverted, whereas in invert_doublet the non-degenerate doublet is inverted.

The main part of the two executables is depicted in Fig. 3. Each measurement corresponds to one gauge configuration that is read from disk into memory. For each of these gauge configurations a number of inversions will be performed.

The sources can be either generated or read in from disk. In the former case the programme can currently generate point sources at random location in space time. In the latter case the name of the source file can be specified in the input file.

The relevant Dirac operator is then inverted on each source and the result is stored on disk. The inversion can be performed with a number of inversion algorithms, such as conjugate gradient (CG), BiCGstab, and others [32]. And optionally even/odd preconditioning as described previously can be used.

\subsubsection{Command line arguments}

The two programmes offer command line options as follows:

- $-\mathrm{h} \mid$ ? prints a help message and exits.

- $-\mathrm{f}$ input file name. The default is hmc. input.

- - o the prefix of the output filenames. The default is output. The code will generate or append to one file called output.para.

\subsubsection{Output}

The program will create a file called output.para with information about the parameters of the run. Of course, also the propagators are stored on disk. The corresponding file names can be influenced via input parameters. The file format is discussed in some detail in Section 4.7.

One particularity of the invert_doublet program is that the propagators written to disk correspond to the two flavour Dirac operator of Eq. (6), i.e.

$$
D_{h}^{\prime}\left(\mu_{\sigma}, \mu_{\delta}\right)=D_{\mathrm{W}} \cdot 1_{f}+i \mu_{\sigma} \tau^{1}+\gamma_{5} \mu_{\delta} \tau^{3},
$$

essentially for compatibility reasons. For the two flavour components written the first is the would be strange component and the second one the would be charm one.

\subsection{Parallelisation}

The whole lattice can be parallelised in up to 4 space-time directions. It is controlled with configure switches, see Section 5.2. The Message Passing Interface (MPI, standard version 1.1) is used to implement the parallelisation. So for compiling the parallel executables a working MPI implementation is needed.

Depending on the number of parallelised space-time directions the $t$-direction, the $t$ - and $x$-direction, the $t$-, $x$ - and $y$-direction or the $t$-, $x$ - and $y$ - and $z$-direction are parallelised.

The number of processors per space direction must be specified at run time, i.e. in the input file. The relevant parameters are NrXProcs, NrYProcs and NrZProcs. The number of processors in time direction is determined by the program automatically. Note that the extension in any direction must divide by the number of processors in this direction.

In case of even/odd preconditioning further constraints have to be fulfilled: the local $L_{z}$ and the local product $L_{t} \times L_{x} \times L_{y}$ must both be even. 


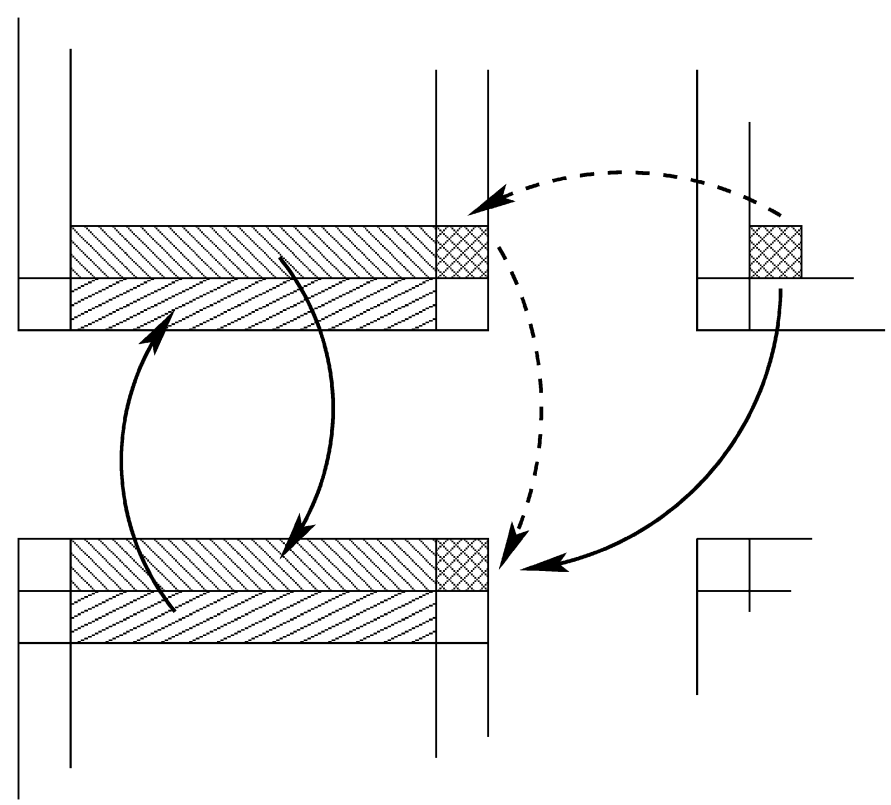

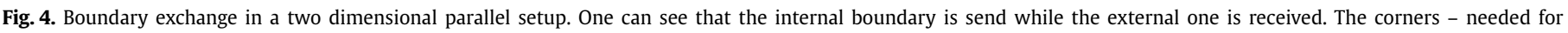
implementing improved gauge actions like the tree-level Symanzik improved gauge action [33] - need a two step procedure.

The communication is organised using boundary buffer, as sketched in Fig. 4. The MPI setup is contained in the file mpi_init.c. The corresponding function must be called at the beginning of a main program just after the parameters are read in, also in case of a serial run. In this function also the various MPI_Datatypes are constructed needed for the exchange of the boundary fields. The routines performing the communication for the various data types are located in files starting with xchange_.

The communication is implemented using different types of MPI functions. One implementation uses the MPI_Sendrecv function to communicate the data. A second one uses non-blocking MPI functions and a third one persistent MPI calls. See the MPI standard for details [34]. On machines with network capable of sending in several directions in parallel the non-blocking version is the most efficient one. The relevant configure switches are - -with-nonblockingmpi and - -with-persistentmpi, the latter of which is only available for the Dirac operator with halfspinor fields, see Section 4.1 .

\section{Description of the individual software components}

\subsection{Dirac operator}

The Dirac operator is the kernel routine of any lattice QCD application, because its inverse is needed for the HMC update procedure and also for computing correlation functions. The inversion is usually performed by means of iterative solvers, like the conjugate gradient algorithm, and hence the repeated application of the Dirac operator to a spinor field is needed. Thus the optimisation of this routine deserves special attention.

At some space-time point $x$ the application of a Wilson type Dirac operator is mainly given by

$$
\phi(x)=\left(m_{0}+4 r+i \mu_{q} \gamma_{5}\right) \psi(x)-\frac{1}{2} \sum_{\mu=1}^{4}\left[U_{x, \mu}\left(r+\gamma_{\mu}\right) \psi(x+a \hat{\mu})+U_{x-a \hat{\mu}, \mu}^{\dagger}\left(r-\gamma_{\mu}\right) \psi(x-a \hat{\mu})\right]
$$

where $r$ is the Wilson parameter, which we set to one in the following. The most computer time consuming part is the nearest neighbour interaction part.

For this part it is useful to observe that

$$
\left(1 \pm \gamma_{\mu}\right) \psi
$$

has only two independent spinor components, the other two follow trivially. So only two of the components need to be computed, then to be multiplied with the corresponding gauge field $U$, and then the other two components are to be reconstructed.

The operation in Eq. (22) must be performed for each space-time point $x$. If the loop over $x$ is performed such that all elements of $\phi$ are accessed sequentially (one output stream), it is clear that the elements in $\psi$ and $U$ cannot be accessed sequentially as well. This non-sequential access may lead to serious performance degradations due to too many cache misses, because modern processing units have only a very limited number of input streams available.

While the $\psi$ field is usually different from one to the next application of the Dirac operator, the gauge field stays often the same for a large number of applications. This is for instance so in iterative solvers, where the Dirac operator is applied $\mathcal{O}(1000)$ times with fixed gauge fields. Therefore it is useful to construct a double copy of the original gauge field sorted such that the elements are accessed exactly in the order needed in the Dirac operator. For the price of additional memory, with this simple change one can obtain large performance improvements, depending on the architecture. The double copy must be updated whenever the gauge field changes. This feature is available in the code at configure time, the relevant switch is - -with-gaugecopy. 


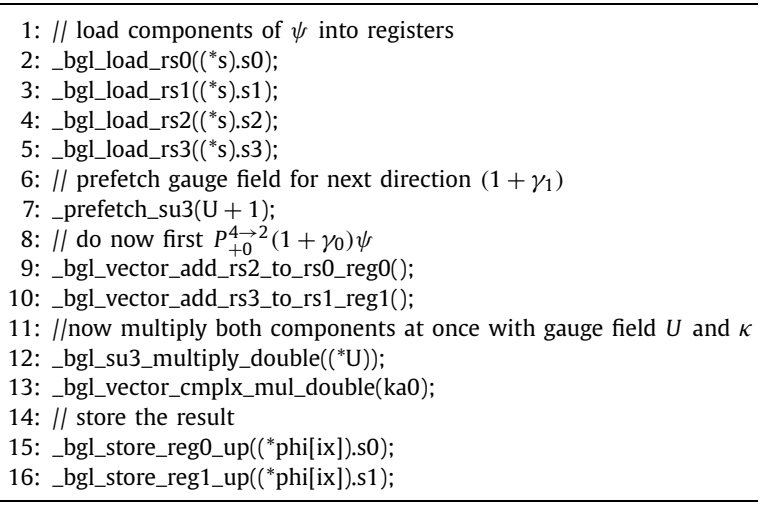

Algorithm 1. $\varphi^{+}=\kappa U P_{+0}^{4 \rightarrow 2}\left(1+\gamma_{0}\right) \psi$.

Above we were assuming that we run sequentially through the resulting spinor field $\phi$. Another possibility is to run sequentially through the source spinor field $\psi$. Moreover, one could split up the operation (22) following the standard trick of introducing intermediate result vectors $\varphi^{ \pm}$with only two spinor components per lattice site. Concentrating on the hopping part only, we would have

$$
\begin{aligned}
& \varphi^{+}(x, \mu)=P_{+\mu}^{4 \rightarrow 2} U_{x, \mu}\left(r+\gamma_{\mu}\right) \psi(x), \\
& \varphi^{-}(x, \mu)=P_{-\mu}^{4 \rightarrow 2}\left(r-\gamma_{\mu}\right) \psi(x) .
\end{aligned}
$$

From $\varphi^{ \pm}$we can then reconstruct the resulting spinor field as

$$
\phi(x)=\sum_{\mu} P_{+\mu}^{2 \rightarrow 4} \varphi^{+}(x+a \hat{\mu}, \mu)+\sum_{\mu} P_{-\mu}^{2 \rightarrow 4} U_{x-a \hat{\mu}, \mu}^{\dagger} \varphi^{-}(x-a \hat{\mu}, \mu) .
$$

Here we denote with $P_{ \pm \mu}^{4 \rightarrow 2}$ the projection to the two independent spinor components for $1 \pm \gamma_{\mu}$ and with $P_{ \pm \mu}^{2 \rightarrow 4}$ the corresponding reconstruction. The half spinor fields $\varphi^{ \pm}$can be interlaced in memory such that $\psi(x)$ as well as $\varphi^{ \pm}(x)$ are always accessed sequentially in memory. The same is possible for the gauge fields, as explained above. So only for $\phi$ we cannot avoid strided access. So far we have only introduced extra fields $\varphi^{ \pm}$, which need to be loaded and stored from and to main memory, and divided the Dirac operator into two steps (23) and (24) which are very balanced with regard to memory bandwidth and floating point operations.

The advantage of this implementation of the Dirac operator comes in the parallel case. In step (23) we need only elements of $\psi(x)$, which are locally available on each node. So this step can be performed without any communication. In between steps (23) and (24) one then needs to communicate part of $\varphi^{ \pm}$, however only half the amount is needed compared to a communication of $\psi$. After the second step there is then no further communication needed. Hence, one can reduce the amount of data to be sent by a factor of two.

There is yet another performance improvement possible with this form of the Dirac operator, this time for the price of precision. One can store the intermediate fields $\varphi^{ \pm}$with reduced precision, e.g. in single precision when the regular spinor fields are in double precision. This will lead to a result with reduced precision, however, in a situation where this is not important, as for instance in the MD update procedure, it reduces the data to be communicated by another factor of two. And the required memory bandwidth is reduced as well. This version of the hopping matrix (currently it is only implemented for the hopping matrix) is available at configure time with the switch - -enable-halfspinor.

The reduced precision version (sloppy precision) is available through the input parameter UseSloppyPrecision. It will be used in the MD update where appropriate. Moreover, it is implemented in the CG iterative solver following the ideas outlined in Ref. [35] for the overlap operator.

The various implementations of the Dirac operator can be found in the file D_psi.c and - as needed for even/odd preconditioning the hopping matrix in the file Hopping_Matrix.c. There are many different versions of these two routines available, each optimised for a particular architecture, e.g. for the Blue Gene/P double hummer processor or the streaming SIMD extensions of modern PC processors (SSE2 and SSE3), see also Ref. [36]. Martin Lüscher has made available his standard C and SSE/SSE2 Dirac operator [37] under the GNU General Public License, which are partly included into the tmLQCD package.

\subsubsection{Blue Gene version}

The IBM PowerPC 450d processor used on the Blue Gene architecture provides a dual FPU, which supports a set of SIMD operations working on 32 special registers useful for lattice QCD. These operations can be accessed using build in functions of the IBM XLC compiler. The file $\operatorname{bg} 1$. $h$ contains all macros relevant for the Blue Gene version of the hopping matrix and the Dirac operator.

A small fraction of half spinor version (see above) is given in Algorithm 1, which represents the operation $\varphi^{+}=\kappa U P_{+0}^{4 \rightarrow 2}\left(1+\gamma_{0}\right) \psi$. After loading the components of $\psi$ into the special registers and prefetching the gauge field for the next direction (in this case $1+\gamma_{1}$ ), $P_{+0}^{4 \rightarrow 2}\left(1+\gamma_{0}\right) \psi$ is performed. It is then important to load the gauge field $U$ only once from memory to registers and multiply both spinor components in parallel.

Finally the result is multiplied with $\kappa$ (which inherits also a phase factor due to the way we implement the boundary conditions, see next sub-section) and stored in memory. 


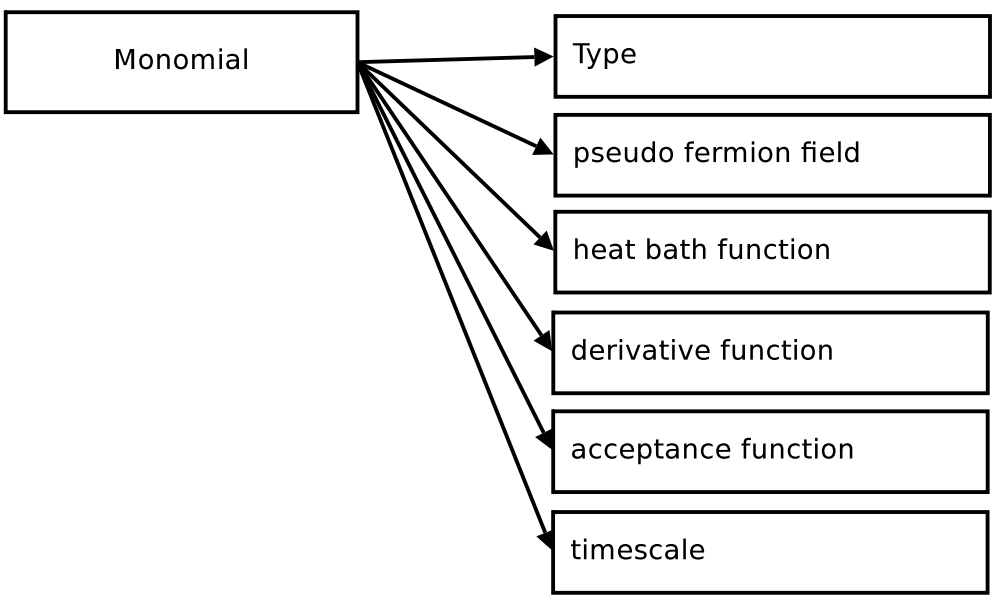

Fig. 5. Data type monomial and its components.

\subsubsection{Boundary conditions}

As discussed previously, we allow for arbitrary phase factors in the boundary conditions of the fermion fields. This is conveniently implemented in the Dirac operator as a phase factor in the hopping term

$$
\sum_{\mu}\left[e^{i \theta_{\mu} \pi / L_{\mu}} U_{x, \mu}\left(r+\gamma_{\mu}\right) \psi(x+a \hat{\mu})+e^{-i \theta_{\mu} \pi / L_{\mu}} U_{x-a \hat{\mu}, \mu}^{\dagger}\left(r-\gamma_{\mu}\right) \psi(x-a \hat{\mu})\right] .
$$

The relevant input parameters are ThetaT, ThetaX, ThetaY, ThetaZ.

\subsection{The HMC update}

We assume in the following that the action to be simulated can be written as

$$
S=S_{\mathrm{G}}+\sum_{i=1}^{N_{\text {monomials }}} S_{\mathrm{PF}_{i}}
$$

and we call - following the CHROMA notation [38] - each term in this sum a monomial. We require that there is exactly one gauge monomial $S_{\mathrm{G}}$ (which we identify with $S_{0}$ in the following) and an arbitrary number of pseudo fermion monomials $S_{\mathrm{PF}_{i}}$.

As a data type every monomial must known how to compute its contribution to the initial Hamiltonian $\mathcal{H}$ at the beginning of each trajectory in the heat-bath step. Then it must know how to compute the derivative with respect to the gauge fields for given gauge field and pseudo fermion field needed for the MD update. And finally there must be a function to compute its contribution to the final Hamiltonian $\mathcal{H}^{\prime}$ as used in the acceptance step.

In addition for each monomial it needs to be known on which timescale it should be integrated. The corresponding data type is sketched in Fig. 5. The general definitions for this data type can be found in the file monomial.c.

There are several sorts of monomials implemented:

- DET: pseudo fermion representation of the (mass degenerate) simple determinant

$$
\operatorname{det}\left(Q^{2}(\kappa)+\mu^{2}\right) \text {. }
$$

- DETRATiO: pseudo fermion representation of the determinant ratio

$$
\operatorname{det}\left(Q^{2}(\kappa)+\mu^{2}\right) / \operatorname{det}\left(Q^{2}\left(\kappa_{2}\right)+\mu_{2}^{2}\right) .
$$

- NDPOLY: polynomial representation of the (possibly non-degenerate) doublet

$$
\left[\operatorname{det}\left(Q_{n d}(\bar{\epsilon}, \bar{\mu})^{2}\right)\right]^{1 / 2} \text {. }
$$

- GAUGE:

$$
\frac{\beta}{3} \sum_{x}\left(c_{0} \sum_{\substack{\mu, \nu=1 \\ 1 \leqslant \mu<\nu}}^{4}\left\{1-\operatorname{Re} \operatorname{Tr}\left(U_{x, \mu, \nu}^{1 \times 1}\right)\right\}+c_{1} \sum_{\substack{\mu, \nu=1 \\ \mu \neq \nu}}^{4}\left\{1-\operatorname{Re} \operatorname{Tr}\left(U_{x, \mu, \nu}^{1 \times 2}\right)\right\}\right) .
$$

The parameter $c_{1}$ can be set in the input file and $c_{0}=1-8 c_{1}$. Note that $c_{1}=0$ corresponds to the Wilson plaquette gauge action.

The corresponding specific functions are defined in the files det_monomial.c, detratio_monomial.c,ndpoly_monomial.c and gauge_monomial.c. Additional monomials can easily be implemented by providing the corresponding functions as discussed above.

The integration scheme is implemented recursively, as exemplified in Algorithm 2 for the leap-frog integration scheme (where we skipped half steps for simplicity). The updateMomenta function simply calls the derivative functions of all monomials that are integrated on timescale $n_{\mathrm{ts}}$ and updates the momenta $P$ according to the time step $\Delta \tau$. 


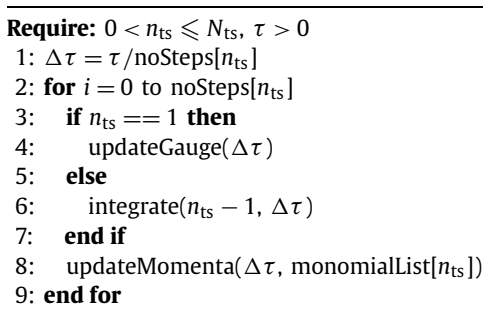

Algorithm 2. Integrate.

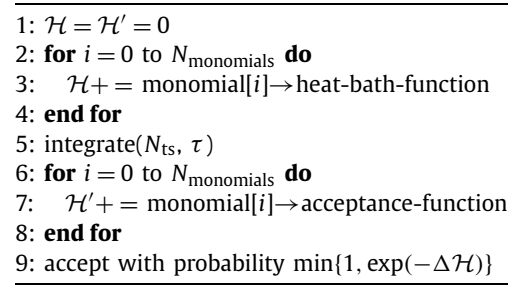

Algorithm 3. MD update.

The recursive scheme for the integration can easily be extended to more involved integration schemes. The details can be found in the file integrator. c. We have implemented the leap-frog and the second order minimal norm [21] integrations schemes. They are named in the input file as LEAPFROG and 2MN, respectively. These two can be mixed on different timescales. In addition we have implemented a position version of the second order minimal norm integration scheme, denoted by 2 MNPOSITION in the input file. The latter must not be mixed with the former two.

The MD update is summarised in Algorithm 3. It computes the initial and final Hamiltonians and calls in between the integration function with the total number of timescales $N_{\mathrm{ts}}$ and the total trajectory length $\tau$.

\subsubsection{Reduced precision in the MD update}

As shortly discussed previously, as long as the integration in the MD update is reversible and area preserving there is large freedom in choosing the integration scheme, but also the operator: it is not necessary to use the Dirac operator here, it can be any approximation to it. This is only useful if the acceptance rate is not strongly affected by such an approximation.

The code provides two possibilities to adapt the precision of the Dirac operator used in the MD update: the first is to reduce the precision in the inversions needed for the force computation. This causes reduced iteration numbers needed for the integration of one trajectory. The relevant input parameter is ForcePrecision available for each monomial. The precision needed in the acceptance and/or heat-bath step can be adjusted separately using AcceptancePrecision. It is advisable to have the acceptance precision always close to machine precision.

The second possibility for influencing the Dirac operator is given by the reduced precision Dirac operator described in Section 4.1, which is switched on with the UseSloppyPrecision input parameter. The two possibilities can also be used in parallel.

Note that one should always test for reversibility violations as explained in Section 4.3 .

\subsubsection{Chronological solver}

The idea of the chronological solver method, or chronological solver guess (CSG) (or similar methods [39]) is to optimise the initial

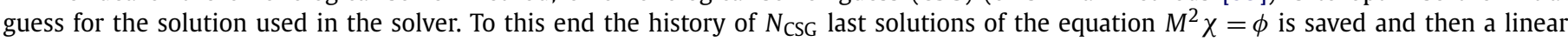
combination of the fields $\chi_{i}$ with coefficients $c_{i}$ is used as an initial guess for the next inversion. $M$ stands for the operator to be inverted and has to be replaced by the different ratios of operators used in this paper.

The coefficients $c_{i}$ are determined by solving

$$
\sum_{i} \chi_{j}^{\dagger} M^{2} \chi_{i} c_{i}=\chi_{j}^{\dagger} \phi
$$

with respect to the coefficients $c_{i}$. This is equivalent to minimising the functional that is minimised by the CG inverter itself.

The downside of this method is that the reversibility violations increase significantly by one or two orders of magnitude in the Hamiltonian when the CSG is switched on and all other parameters are kept fixed. Therefore one has to adjust the residues in the solvers, which increases the number of matrix vector multiplications again. Our experience is that the methods described in the previous subsection are more effective in particular in the context of multiple time scale integration, because the CSG is most effective for small values of $\Delta \tau$.

The relevant input parameter is the CSGHistory parameter available for DET and DETRATIO monomials. Setting it to zero means no chronological solver, otherwise this parameter specifies the number of last solutions $N_{\text {CSG }}$ to be saved.

\subsection{Online measurements}

The HMC program includes the possibility to perform a certain number of measurements after every trajectory online, whether or not the configuration is stored on disk. Some of those are performed per default, namely all that are written to the output file output. data: 
(1) the plaquette expectation value, defined as:

$$
\langle P\rangle=\frac{1}{6 V} \sum_{\substack{\mu, \nu=1 \\ 1 \leqslant \mu<\nu}}^{4} \operatorname{Re} \operatorname{Tr}\left(U_{x, \mu, \nu}^{1 \times 1}\right),
$$

where $V$ is the global lattice volume;

(2) the rectangle expectation value, defined as:

$$
\langle R\rangle=\frac{1}{12 V} \sum_{\substack{\mu, \nu=1 \\ \mu \neq \nu}}^{4} \operatorname{Re} \operatorname{Tr}\left(U_{x, \mu, \nu}^{1 \times 2}\right) .
$$

(3) $\Delta \mathcal{H}=\mathcal{H}^{\prime}-\mathcal{H}$ and $\exp (-\Delta \mathcal{H})$.

See the overview section for details about the output. data file. These observables all come with no extra computational cost.

Optionally, other online measurements can be performed, which - however - need in general extra inversions of the Dirac operator. First of all the computation of certain correlation functions is implemented. They need one extra inversion of the Dirac operator, as discussed in Ref. [40], using the one-end-trick. Define a stochastic source $\xi$ as follows

$$
\lim _{R \rightarrow \infty}\left[\xi_{i}^{*} \xi_{j}\right]=\delta_{i j}, \quad \lim _{R \rightarrow \infty}\left[\xi_{i} \xi_{j}\right]=0 .
$$

Here $R$ labels the number of samples and $i$ all other degrees of freedom. Then

$$
\left[\phi_{i}^{r *} \phi_{j}^{r}\right]_{R}=M_{i k}^{-1 *} \cdot M_{j k}^{-1}+\text { noise }
$$

if $\phi$ was computed from

$$
\phi_{j}^{r}=M_{j k}^{-1} \xi_{k}^{r} \text {. }
$$

Having in mind the $\gamma_{5}$-hermiticity property of the Wilson and Wilson twisted mass Dirac propagator $G_{u, d}$, i.e.

$$
G_{u}(x, y)=\gamma_{5} G_{d}(y, x)^{\dagger} \gamma_{5}
$$

it is clear that Eq. (27) can be used to evaluate

$$
C_{\pi}(t)=\left\langle\operatorname{Tr}\left[G_{u}(0, t) \gamma_{5} G_{d}(t, 0) \gamma_{5}\right]\right\rangle=\left\langle\operatorname{Tr}\left[G_{u}(0, t) G_{u}(0, t)^{\dagger}\right]\right\rangle
$$

with only one inversion. But, even if the one gamma structure at the source is fixed to be $\gamma_{5}$ due to the $\gamma_{5}$-hermiticity trick, we are still free to insert any $\gamma$-structure $\Gamma$ at the source, i.e. we can evaluate any correlation function of the form

$$
C_{P \Gamma}(t)=\left\langle\operatorname{Tr}\left[G_{u}(0, t) \gamma_{5} G_{d}(t, 0) \Gamma\right]\right\rangle=\left\langle\operatorname{Tr}\left[G_{u}(0, t) G_{u}(0, t)^{\dagger} \gamma_{5} \Gamma\right]\right\rangle .
$$

Useful combinations of correlation functions are $\langle P P\rangle,\langle P A\rangle$ and $\langle P V\rangle$, with

$$
P^{\alpha}=\bar{\chi} \gamma_{5} \frac{\tau^{\alpha}}{2} \chi, \quad V_{\mu}^{\alpha}=\bar{\chi} \gamma_{\mu} \frac{\tau^{\alpha}}{2} \chi, \quad A_{\mu}^{\alpha}=\bar{\chi} \gamma_{5} \gamma_{\mu} \frac{\tau^{\alpha}}{2} \chi
$$

From $\langle P P\rangle$ one can extract the pseudo scalar mass, and - in the twisted mass case - the pseudo scalar decay constant. $\langle P A\rangle$ can be used together with $\langle P P\rangle$ to extract the so called PCAC quark mass and $\langle P V\rangle$ to measure the renormalisation constant $Z_{V}$. For details we refer the reader to Ref. [40].

These online measurements are controlled with the two following input parameters: PerformonlineMeasurements to switch them on or off and to specify the frequency onlineMeasurementsFreq. The three correlation functions are saved in files named onlinemeas.n, where $\mathrm{n}$ is the trajectory number. Every file contains five columns, specifying the type, the operator type and the Euclidean time $t$. The last two columns are the values of the correlation function itself, $C(t)$ and $C(-t)$, respectively. The type is equal to 1,2 or 6 for the $\langle P P\rangle$, the $\langle P A\rangle$ and the $\langle P V\rangle$ correlation functions. The operator type is for online measurements always equal to 1 for local source and sink (no smearing of any kind), and the time runs from 0 to $T / 2$. Hence, $C(-t)=C(T-t)$. $C(-0)$ and $C(-T / 2)$ are set to zero for convenience.

In addition to correlation functions also the minimal and the maximal eigenvalues of the $\left(\gamma_{5} D\right)^{2}$ can be measured.

An online measurement not related to physics, but related to the algorithm are checks of reversibility violations. The HMC algorithm is exact if and only if the integration scheme is reversible. On a computer with finite precision this is only guaranteed up to machine precision. These violations can be estimated by integrating one trajectory forward and then backward in Monte Carlo time. The difference $\delta \Delta \mathcal{H}$ among the original Hamiltonian $\mathcal{H}$ and the final one $\mathcal{H}^{\prime \prime}$ after integrating back can serve as one measure for those violations, another one is provided by the difference among the original gauge field $U$ and the final one $U^{\prime \prime}$

$$
\delta \Delta U=\frac{1}{12 V} \sum_{x, \mu} \sum_{i, j}\left(U_{x, \mu}-U_{x, \mu}^{\prime \prime}\right)_{i, j}^{2}
$$

where we indicate with the $\delta \Delta$ that this is obtained after integrating a trajectory forward and backward in time. The results for $\delta \Delta \mathcal{H}$ and $\delta \Delta U$ are stored in the file return_check. data. The relevant input parameters are Reversibilitycheck and ReversibilityCheckInterval. 


\subsection{Iterative solver and eigensolver}

There are several iterative solvers implemented in the tmLQCD package for solving

$$
D \chi=\phi
$$

for $\chi$. The minimal residual (MR), the conjugate gradient (CG), the conjugate gradient squared (CGS), the generalised minimal residual (GMRES), the generalised conjugate residual (GCR) and the stabilised bi-conjugate gradient (BiCGstab). For details regarding these algorithms we refer to Refs. [32,41].

For the hmc_tm executable only the CG and the BiCGstab solvers are available, while all the others can be used in the invert executables. Most of them are both available with and without even/odd preconditioning. For a performance comparison we refer to Refs. [35,42].

The stopping criterion is implemented in two ways: the first is an absolute stopping criterion, i.e. the solver is stopped when the squared norm of the residual vector (depending on the solver this might be the iterated residual or the real residual) fulfils

$$
\|r\|^{2}<\epsilon^{2}
$$

The second is relative to the source vector, i.e.

$$
\frac{\|r\|^{2}}{\|\phi\|^{2}}<\epsilon^{2} \text {. }
$$

The value of $\epsilon^{2}$ and the choice of relative or absolute precision can be influenced via input parameters.

The reduced precision Dirac operator, as discussed in Section 4.1, is available for the CG solver. In the CG solver the full precision Dirac operator is only required at the beginning of the CG search, because the relative size of the contribution to the resulting vector decreases with the number of iterations. Thus, as soon as a certain precision is achieved in the CG algorithm we can switch to the reduced precision Dirac operator without spoiling the precision of the final result. We switch to the lower precision operator at a precision of $\sqrt{\epsilon}$ in the CG search, when aiming for a final precision of $\epsilon<1$.

We note that in principle any combination of using reduced precision in one of the ways described in this paper is possible. However, one should always check that the true residual is as small as expected in case of an inversion and that the reversibility violations are small in case of a HMC simulation.

The eigensolver used to compute the eigenvalues (and vectors) of $\left(\gamma_{5} D\right)^{2}$ is the so called Jacobi-Davidson method [43,44]. For a discussion for the application of this algorithm to lattice QCD we refer again to Refs. [35,42].

All solver related files can be found in the sub-directory solver. Note that there are a few more solvers implemented which are, however, in an experimental status.

\subsection{Stout smearing}

Smearing techniques have become an important tool to reduce ultraviolet fluctuations in the gauge fields. One of those techniques, coming with the advantage of being usable in the MD update, is usually called stout smearing [45].

The $(n+1)$ th level of stout smeared gauge links is obtained iteratively from the $n$th level by

$$
U_{\mu}^{(n+1)}(x)=e^{i Q_{\mu}^{(n)}(x)} U_{\mu}^{(n)}(x)
$$

We refer to the unsmeared ("thin") gauge field as $U_{\mu} \equiv U_{\mu}^{(0)}$. The SU(3) matrices $Q_{\mu}$ are defined via the staples $C_{\mu}$ :

$$
\begin{aligned}
& Q_{\mu}^{(n)}(x)=\frac{i}{2}\left[U_{\mu}^{(n)}(x) C_{\mu}^{(n)^{\dagger}}(x)-\text { h.c. }\right]-\frac{i}{6} \operatorname{Tr}\left[U_{\mu}^{(n)}(x) C_{\mu}^{(n)^{\dagger}}(x)-\text { h.c. }\right], \\
& C_{\mu}^{(n)}=\sum_{\nu \neq \mu} \rho_{\mu \nu}\left(U_{\nu}^{(n)}(x) U_{\mu}^{(n)}(x+\hat{v}) U_{\nu}^{(n)}{ }^{\dagger}(x+\hat{\mu})+U_{v}^{(n)^{\dagger}}(x-\hat{v}) U_{\mu}^{(n)}(x-\hat{v}) U_{v}^{(n)}(x-\hat{v}+\hat{\mu})\right),
\end{aligned}
$$

where in general $\rho_{\mu v}$ is the smearing matrix. In the tmLQCD package we have only implemented isotropic 4-dimensional smearing, i.e., $\rho_{\mu \nu}=\rho$.

Currently stout smearing is only implemented for the invert executables. I.e. the gauge field can be stout smeared at the beginning of an inversion. The input parameters are UseStoutSmearing, StoutRho and StoutNoIterations.

\subsection{Random number generator}

The random number generator used in the code is the one proposed by Martin Lüscher and usually known under the name RANLUX [46]. A single and double precision implementation was made available by the author under the GNU General Public License and can be downloaded [47]. For convenience it is also included in the tmLQCD package.

\subsection{IO formats}

In this final subsection we specify the IO formats used to store gauge configurations, propagators and sources to disk. 


\subsubsection{Gauge configurations}

For gauge configurations we use the International Lattice Data Grid (ILDG) standard as specified in Refs. [48,49]. As the lime packaging library [50] and ILDG standard allow additional - not required - records to be stored within the file, we currently add the following two records for convenience:

(1) $x l f$-info: useful information about the gauge configuration, such as the plaquette value, and about the run and the algorithm and the code version used to generate it.

(2) scidac-checksum: SCIDAC checksum of the gauge configuration. For the specification see [51].

The gauge configurations can be written to disk either in single or double precision. The relevant input parameter is GaugeConfigWritePrecision. On reading the precision is determined automatically.

Note that the gauge configuration does not depend on the particular choice of the $\gamma$-matrices.

\subsubsection{Propagators}

We note at the beginning, that we do not use different IO formats for source or sink fermion fields. They are both stored using the same lime records. The meta-data stored in the same lime-packed file is supposed to clarify all other things. It is also important to realise that the propagator depends on the $\gamma$-matrix convention used in the Dirac operator. For our convention see Appendix A.

Here we mainly concentrate on storing propagators (sink). The file can contain only sinks, or both, source and sink. We (plan to) support four different formats

(1) (arbitrary number of) sink, no sources

(2) (arbitrary number of) source/sink pairs

(3) one source, 12 sink

(4) one source, 4 sink

This is very similar to the formats in use in parts of the US lattice community. We adopt the SCIDAC checksum [51] for the binary data.

Source and sink binary data has to be in a separate lime record. The order in one file for the four formats mentioned above is supposed to be

(1) sink, no sources: -

(2) source/sink pairs: first source, then sink

(3) one source, 12 sink: first source, then 12 sinks

(4) one source, 4 sink: first source, then 4 sinks

The first two types are by now supported in the tmLQCD package. In the future the other two might follow. All fermion field files must have a record indicating its type. The record itself is of type propagator-type and the record has a single entry (ASCII string) which contains one of

- DiracFermion_Sink

- DiracFermion_Source_Sink_Pairs

- DiracFermion_ScalarSource_TwelveSink

- DiracFermion_ScalarSource_FourSink

Those strings are also used in the input files for the input parameter PropagatorType. The binary data corresponding to one Dirac fermion field (source or sink) is then stored with at least two (three) records. The first is of type etmc-propagator-format and contains the following information:

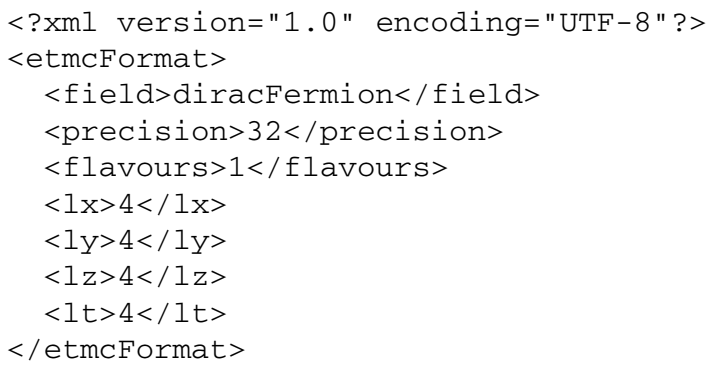

The flavours entry must be set to 1 for a one flavour propagator (flavour diagonal case) and to 2 for a two flavour propagator (flavour non-diagonal 2-flavour operator). In the former case there follows one record of type scidac-binary-data, which is identical to the SCIDAC format, containing the fermion field. In the latter case there follow two of such records, the first of which is the upper flavour. To be precise, lets call the two flavours $s$ (strange) and $c$ (charm). Then we always store the $s$ component first and then the $c$ component.

The indices (time, space, spin, colour) in the binary data scidac-binary-data are in the following order:

$$
t, z, y, x, s, c,
$$


where $t$ is the slowest and colour the fastest running index. The binary data is stored big-endian and either in single or in double precision, depending on the precision entry in the etmc-propagator-format record.

In addition we store an additional record called inverter-info with useful information about the inversion precision, the physical parameters and the code version.

\subsubsection{Source fields}

Source fields are, as mentioned before, stored with the same binary data format. There are again several types of source files possible:

- DiracFermion_Source

- DiracFermion_ScalarSource

- DiracFermion_FourScalarSource

- DiracFermion_TwelveScalarSource

This type is stored in a record called source-type in the lime file. There might be several sources stored within the same file. We add a format record etmc-source-format looking like

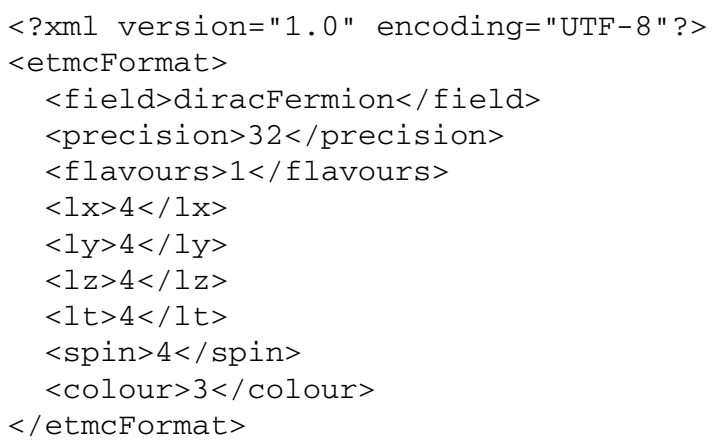

with obvious meaning for every scidac-binary-data record within the lime packed file. This format record also allows to store a subset of the whole field, e.g. a time-slice.

\section{Installation instructions}

The software ships with a GNU autoconf environment and a configure script, which will generate GNU Makefiles to build the programmes. It is supported and recommended to configure and build the executables in a separate build directory. This also allows to have several builds with different options from the same source code directory.

\subsection{Prerequisites}

In order to compile the programmes the LAPACK [52] library (Fortran version) needs to be installed. In addition it must be known which linker options are needed to link against LAPACK, e.g. -Lpath-to-lapack -1lapack -1blas. Also a the latest version (tested is version 1.2.3) of C-LIME [50] must be available, which is used as a packaging scheme to read and write gauge configurations and propagators to files.

\subsection{Configuring the tmLQCD package}

In order to get a simple configuration of the hmc package it is enough to just type

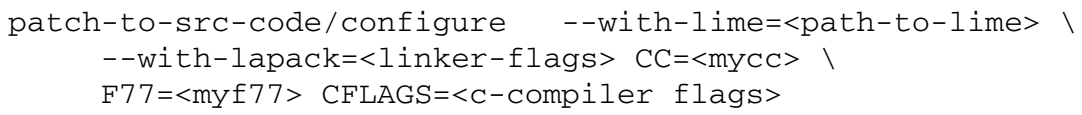

in the build directory. If CC, F77 and CFLAGS are not specified, configure will guess them.

The code was successfully compiled and run at least on the following platforms: i686 and compatible, x64 and compatible, IBM Regatta systems, IBM Blue Gene/L, IBM Blue Gene/P, SGI Altix and SGI PC clusters and powerpc clusters.

The configure script accepts certain options to influence the building procedure. One can get an overview over all supported options with configure - -help. There are enable|disable options switching on and off optional features and with|without switches usually related to optional packages. In the following we describe the most important of them (check configure - -help for the defaults and more options):

- - -enable-mpi:

This option switches on the support for MPI. On certain platforms it automatically chooses the correct parallel compiler or searches for a command mpicc in the search path.

- - -enable-p4:

Enable the use of special Pentium 4 instruction set and cache management. 
Table 1

Parameter and results for three sample input files as provided with the code.

\begin{tabular}{|c|c|c|c|}
\hline & $\mathrm{TR}_{0}$ & $\mathrm{TR}_{1}$ & $\mathrm{TR}_{2}$ \\
\hline input-file & sample-hmc0.input & sample-hmc2.input & sample-hmc3.input \\
\hline$L^{3} \times T$ & $4^{3} \times 4$ & $4^{3} \times 4$ & $4^{3} \times 4$ \\
\hline$S_{G}$ & Wilson & TlSym & Iwasaki \\
\hline$\beta$ & 6.0 & 3.3 & 1.95 \\
\hline$\kappa$ & 0.177 & 0.17 & 0.163260 \\
\hline $2 \kappa \mu_{q}$ & 0.177 & 0.01 & 0.002740961 \\
\hline $2 \kappa \bar{\mu}$ & - & 0.1105 & - \\
\hline $2 \kappa \bar{\epsilon}$ & - & 0.0935 & - \\
\hline$\langle P\rangle$ & $0.62457(7)$ & $0.53347(17)$ & $0.5951(2)$ \\
\hline$\langle R\rangle$ & - & $0.30393(22)$ & $0.3637(3)$ \\
\hline
\end{tabular}

- - -enable-opteron:

Enable the use of special opteron instruction set and cache management.

- - - enable-sse2:

Enable the use of SSE2 instruction set. This is a huge improvement on Pentium 4 and equivalent systems.

- - - enable-sise3:

Enable the use of SSE3 instruction set. This will give another $20 \%$ of speedup when compared to only SSE2. However, only a few processors are capable of SSE3.

- - -enable-gaugecopy:

See Section 4.1 for details on this option. It will increase the memory requirement of the code.

- - -enable-halfspinor:

If this option is enabled the Dirac operator using half spinor fields is used. See Section 4.1 for details. If this feature is switched on, also the gauge copy feature is switched on automatically.

- - -with-mpidimension=n:

This option has only effect if the code is configured for MPI usage. The number of parallel directions can be specified. $1,2,3$ and 4 dimensional parallelisation is supported.

- - -with-lapack="<linker flags $>$ ":

the code requires lapack to be linked. All linker flags necessary to do so must be specified here. Note that LIBS=" . . " works similar.

- - -with-limedir=<dir>:

Tells configure where to find the lime package, which is required for the build of the HMC. It is used for the ILDG file format.

The configure script will guess at the very beginning on which platform the build is done. In case this fails or a cross compilation must be performed please use the option - -host=HOST. For instance in order to compile for the BG/P one needs to specify - -host=ppc-ibm-bprts - -build=ppc64-ibm-linux.

For certain architectures like the Blue Gene systems there are README. arch files in the top source directory with example configure calls.

\subsection{Building and installing}

After successfully configuring the package the code can be build by simply typing make in the build directory. This will compile the standard executables. Typing make install will copy these executables into the install directory. The default install directory is \$HOME/bin, which can be influenced e.g. with the - -prefix option to configure.

\section{Test run description}

The source code ships with a number of sample input files. They are located in the sample-input sub-directory. They are small volume $V=4^{4}$ test runs designated to measure for instance the average plaquette values.

Such a test-run can be performed for instance on a scalar machine by typing

./hmc_tm -f sample-hmc0.input.

Depending on the environment you are running in, you may need to adjust the input parameters to match the maximal run-time and so on. The expected average plaquette values are quoted in Table 1 and also in the sample input files.

\subsection{Benchmark executable}

Another useful test executable is a benchmark code. It can be build by typing make benchmark and it will, when run, measure the performance of the Dirac operator. It can be run in the serial or parallel case. It reads its input from a file benchmark. input and the relevant input parameters are the following:

$\mathrm{L}=4$

$\mathrm{T}=4$

NrXProcs $=2$

NrYProcs $=2$ 
NrZProcs $=2$

UseEvenodd = yes

UseSloppyPrecision $=$ no

In case of even/odd preconditioning the performance of the hopping matrix is evaluated, in case of no even/odd the performance of the Dirac operator. The important part of the output of the code is as follows

$[\ldots]$

(1429 Mflops [64 bit arithmetic])

communication switched off

(2592 Mflops [64 bit arithmetic])

The size of the package is 36864 Byte

The bandwidth is $662.91+662.91 \mathrm{MB} / \mathrm{sec}$

The bandwidth is not measured directly but computed from the performance difference among with and without communication and the package size. In case of a serial run the output is obviously reduced.

\section{Acknowledgements}

This code was started based on a HMC implementation for Wilson fermions with mass preconditioning kindly provided by Martin Hasenbusch. Many discussions with, and contributions by Rémi Baron, Thomas Chiarappa, Albert Deuzeman, Roberto Frezzotti, Martin Hasenbusch, Gregorio Herdoiza, Marina Marinkovic, Craig McNeile, Istvan Montvay, Andreas Nube, David Palao, Siebren Reker, Andrea Shindler, Jan Volkholz and Urs Wenger are gratefully acknowledged. We thank Peter Boyle for useful discussions on the efficient implementation of the Dirac operator.

\section{Appendix A. $\gamma$ and Pauli matrices}

In the following we specify our conventions for $\gamma$ - and Pauli-matrices.

\section{A.1. $\gamma$-matrices}

We use the following convention for the Dirac $\gamma$-matrices:

$$
\begin{aligned}
\gamma_{0}=\left(\begin{array}{cccc}
0 & 0 & -1 & 0 \\
0 & 0 & 0 & -1 \\
-1 & 0 & 0 & 0 \\
0 & -1 & 0 & 0
\end{array}\right), & \gamma_{1}=\left(\begin{array}{cccc}
0 & 0 & 0 & -i \\
0 & 0 & -i & 0 \\
0 & +i & 0 & 0 \\
+i & 0 & 0 & 0
\end{array}\right), \\
\gamma_{2}=\left(\begin{array}{cccc}
0 & 0 & 0 & -1 \\
0 & 0 & +1 & 0 \\
0 & +1 & 0 & 0 \\
-1 & 0 & 0 & 0
\end{array}\right), & \gamma_{3}=\left(\begin{array}{cccc}
0 & 0 & -i & 0 \\
0 & 0 & 0 & +i \\
+i & 0 & 0 & 0 \\
0 & -i & 0 & 0
\end{array}\right) .
\end{aligned}
$$

In this representation $\gamma_{5}$ is diagonal and reads

$$
\gamma_{5}=\left(\begin{array}{cccc}
+1 & 0 & 0 & 0 \\
0 & +1 & 0 & 0 \\
0 & 0 & -1 & 0 \\
0 & 0 & 0 & -1
\end{array}\right)
$$

\section{A.2. Pauli-matrices}

For the Pauli-matrices acting in flavour space we use the following convention:

$$
1_{f}=\left(\begin{array}{ll}
1 & 0 \\
0 & 1
\end{array}\right), \quad \tau^{1}=\left(\begin{array}{ll}
0 & 1 \\
1 & 0
\end{array}\right), \quad \tau^{2}=\left(\begin{array}{cc}
0 & -i \\
i & 0
\end{array}\right), \quad \tau^{3}=\left(\begin{array}{cc}
1 & 0 \\
0 & -1
\end{array}\right) .
$$

\section{Appendix B. Even/odd preconditioning}

\section{B.1. HMC update}

In this section we describe how even/odd [29,30] preconditioning can be used in the HMC algorithm in presence of a twisted mass term. Even/odd preconditioning is implemented in the tmLQCD package in the HMC algorithm as well as in the inversion of the Dirac operator, and can be used optionally. 
We start with the lattice fermion action in the hopping parameter representation in the $\chi$-basis written as

$$
\begin{aligned}
S[\chi, \bar{\chi}, U] & =\sum_{x}\left\{\bar{\chi}(x)\left[1+2 i \kappa \mu \gamma_{5} \tau^{3}\right] \chi(x)-\kappa \bar{\chi}(x) \sum_{\mu=1}^{4}\left[U(x, \mu)\left(r+\gamma_{\mu}\right) \chi(x+a \hat{\mu})+U^{\dagger}(x-a \hat{\mu}, \mu)\left(r-\gamma_{\mu}\right) \chi(x-a \hat{\mu})\right]\right\} \\
& \equiv \sum_{x, y} \bar{\chi}(x) M_{x y} \chi(y)
\end{aligned}
$$

similar to Eq. (4). For convenience we define $\tilde{\mu}=2 \kappa \mu$. Using the matrix $M$ one can define the hermitian (two flavour) operator:

$$
Q \equiv \gamma_{5} M=\left(\begin{array}{cc}
Q^{+} & \\
& Q^{-}
\end{array}\right)
$$

where the sub-matrices $Q^{ \pm}$can be factorised as follows (Schur decomposition):

$$
\begin{aligned}
Q^{ \pm} & =\gamma_{5}\left(\begin{array}{cc}
1 \pm i \tilde{\mu} \gamma_{5} & M_{e o} \\
M_{o e} & 1 \pm i \tilde{\mu} \gamma_{5}
\end{array}\right)=\gamma_{5}\left(\begin{array}{cc}
M_{e e}^{ \pm} & M_{e o} \\
M_{o e} & M_{o o}^{ \pm}
\end{array}\right) \\
& =\left(\begin{array}{ll}
\gamma_{5} M_{e e}^{ \pm} & 0 \\
\gamma_{5} M_{o e} & 1
\end{array}\right)\left(\begin{array}{cc}
1 & \left(M_{e e}^{ \pm}\right)^{-1} M_{e o} \\
0 & \gamma_{5}\left(M_{o o}^{ \pm}-M_{o e}\left(M_{e e}^{ \pm}\right)^{-1} M_{e o}\right)
\end{array}\right) .
\end{aligned}
$$

Note that $\left(M_{e e}^{ \pm}\right)^{-1}$ can be computed to be

$$
\left(1 \pm i \tilde{\mu} \gamma_{5}\right)^{-1}=\frac{1 \mp i \tilde{\mu} \gamma_{5}}{1+\tilde{\mu}^{2}}
$$

Using $\operatorname{det}(Q)=\operatorname{det}\left(Q^{+}\right) \operatorname{det}\left(Q^{-}\right)$the following relation can be derived

$$
\begin{aligned}
& \operatorname{det}\left(Q^{ \pm}\right) \propto \operatorname{det}\left(\hat{Q}^{ \pm}\right) \\
& \hat{Q}^{ \pm}=\gamma_{5}\left(M_{o o}^{ \pm}-M_{o e}\left(M_{e e}^{ \pm}\right)^{-1} M_{e o}\right)
\end{aligned}
$$

where $\hat{Q}^{ \pm}$is only defined on the odd sites of the lattice. In the HMC algorithm the determinant is stochastically estimated using pseudo fermion fields $\phi_{0}$ :

$$
\begin{aligned}
& \operatorname{det}\left(\hat{Q}^{+} \hat{Q}^{-}\right)=\int \mathcal{D} \phi_{o} \mathcal{D} \phi_{o}^{\dagger} \exp \left(-S_{\mathrm{PF}}\right), \\
& S_{\mathrm{PF}} \equiv \phi_{o}^{\dagger}\left(\hat{\mathrm{Q}}^{+} \hat{\mathrm{Q}}^{-}\right)^{-1} \phi_{o},
\end{aligned}
$$

where the fields $\phi_{0}$ are defined only on the odd sites of the lattice. In order to compute the force corresponding to the effective action $S_{\mathrm{PF}}$ we need the variation of $S_{\mathrm{PF}}$ with respect to the gauge fields (using $\delta\left(A^{-1}\right)=-A^{-1} \delta A A^{-1}$ ):

$$
\begin{aligned}
\delta S_{\mathrm{PF}} & =-\left[\phi_{0}^{\dagger}\left(\hat{\mathrm{Q}}^{+} \hat{\mathrm{Q}}^{-}\right)^{-1} \delta \hat{\mathrm{Q}}^{+}\left(\hat{\mathrm{Q}}^{+}\right)^{-1} \phi_{0}+\phi_{0}^{\dagger}\left(\hat{\mathrm{Q}}^{-}\right)^{-1} \delta \hat{\mathrm{Q}}^{-}\left(\hat{\mathrm{Q}}^{+} \hat{\mathrm{Q}}^{-}\right)^{-1} \phi_{0}\right] \\
& =-\left[X_{0}^{\dagger} \delta \hat{\mathrm{Q}}^{+} Y_{0}+Y_{0}^{\dagger} \delta \hat{\mathrm{Q}}^{-} X_{0}\right]
\end{aligned}
$$

with $X_{0}$ and $Y_{0}$ defined on the odd sides as

$$
X_{0}=\left(\hat{Q}^{+} \hat{Q}^{-}\right)^{-1} \phi_{0}, \quad Y_{o}=\left(\hat{Q}^{+}\right)^{-1} \phi_{0}=\hat{Q}^{-} X_{0},
$$

where $\left(\hat{Q}^{ \pm}\right)^{\dagger}=\hat{Q}^{\mp}$ has been used. The variation of $\hat{Q}^{ \pm}$reads

$$
\delta \hat{Q}^{ \pm}=\gamma_{5}\left(-\delta M_{o e}\left(M_{e e}^{ \pm}\right)^{-1} M_{e o}-M_{o e}\left(M_{e e}^{ \pm}\right)^{-1} \delta M_{e o}\right),
$$

and one finds

$$
\begin{aligned}
\delta S_{\mathrm{PF}} & =-\left(X^{\dagger} \delta Q^{+} Y+Y^{\dagger} \delta Q^{-} X\right) \\
& =-\left(X^{\dagger} \delta Q^{+} Y+\left(X^{\dagger} \delta Q^{+} Y\right)^{\dagger}\right)
\end{aligned}
$$

where $X$ and $Y$ are now defined over the full lattice as

$$
X=\left(\begin{array}{c}
-\left(M_{e e}^{-}\right)^{-1} M_{e o} X_{o} \\
X_{o}
\end{array}\right), \quad Y=\left(\begin{array}{c}
-\left(M_{e e}^{+}\right)_{Y_{o}}^{-1} M_{e o} Y_{o} \\
Y_{o}
\end{array}\right) .
$$

In addition $\delta Q^{+}=\delta Q^{-}, M_{e o}^{\dagger}=\gamma_{5} M_{o e} \gamma_{5}$ and $M_{o e}^{\dagger}=\gamma_{5} M_{e o} \gamma_{5}$ have been used. Since the bosonic part is quadratic in the $\phi_{0}$ fields, the $\phi_{0}$ are generated at the beginning of each molecular dynamics trajectory with

$$
\phi_{0}=\hat{Q}^{+} r_{o}
$$

where $r_{o}$ is a random spinor field taken from a Gaussian distribution with norm one. 
B.1.1. Mass non-degenerate flavour doublet

Even/odd preconditioning can also be implemented for the mass non-degenerate flavour doublet Dirac operator $D_{h}$ Eq. (5). Denoting

$$
Q^{h}=\gamma_{5} D_{h}
$$

the even/odd decomposition is as follows

$$
\begin{aligned}
Q^{h} & =\left(\begin{array}{cc}
\left(\gamma_{5}+i \bar{\mu} \tau^{3}-\bar{\epsilon} \tau^{1}\right) & Q_{e o}^{h} \\
Q_{o e}^{h} & \left(\gamma_{5}+i \bar{\mu} \tau^{3}-\bar{\epsilon} \tau^{1}\right)
\end{array}\right) \\
& =\left(\begin{array}{cc}
Q_{e e}^{h} & 0 \\
Q_{o e}^{h} & 0
\end{array}\right) \cdot\left(\begin{array}{cc}
1 & \left(Q_{e e}^{h}\right)^{-1} Q_{e o} \\
0 & Q_{o o}^{h}
\end{array}\right)
\end{aligned}
$$

where $Q_{o o}^{h}$ is given in flavour space by

$$
Q_{o o}^{h}=\gamma_{5}\left(\begin{array}{cc}
1+i \bar{\mu} \gamma_{5}-\frac{M_{o e}\left(1-i \bar{\mu} \gamma_{5}\right) M_{e o}}{1+\bar{\mu}^{2}-\bar{\epsilon}^{2}} & -\bar{\epsilon}\left(1+\frac{M_{o e} M_{e o}}{1+\bar{\mu}^{2}-\bar{\epsilon}^{2}}\right) \\
-\bar{\epsilon}\left(1+\frac{M_{o e} M_{e o}}{1+\bar{\mu}^{2}-\bar{\epsilon}^{2}}\right) & 1-i \bar{\mu} \gamma_{5}-\frac{M_{o e}\left(1-i \bar{\mu} \gamma_{5}\right) M_{e o}}{1+\bar{\mu}^{2}-\bar{\epsilon}^{2}}
\end{array}\right)
$$

with the previous definitions of $M_{e o}$ etc. The implementation for the PHMC is very similar to the mass degenerate HMC case.

\section{B.2. Inversion}

In addition to even/odd preconditioning in the HMC algorithm as described above, it can also be used to speed up the inversion of the fermion matrix. Due to the factorisation (B.3) the full fermion matrix can be inverted by inverting the two matrices appearing in the factorisation

$$
\left(\begin{array}{ll}
M_{e e}^{ \pm} & M_{e o} \\
M_{o e} & M_{o o}^{ \pm}
\end{array}\right)^{-1}=\left(\begin{array}{cc}
1 & \left(M_{e e}^{ \pm}\right)^{-1} M_{e o} \\
0 & \left(M_{o o}^{ \pm}-M_{o e}\left(M_{e e}^{ \pm}\right)^{-1} M_{e o}\right)
\end{array}\right)^{-1}\left(\begin{array}{cc}
M_{e e}^{ \pm} & 0 \\
M_{o e} & 1
\end{array}\right)^{-1}
$$

The two factors can be simplified as follows:

$$
\left(\begin{array}{ll}
M_{e e}^{ \pm} & 0 \\
M_{o e} & 1
\end{array}\right)^{-1}=\left(\begin{array}{cc}
\left(M_{e e}^{ \pm}\right)^{-1} & 0 \\
-M_{o e}\left(M_{e e}^{ \pm}\right)^{-1} & 1
\end{array}\right)
$$

and

$$
\left(\begin{array}{cc}
1 & \left(M_{e e}^{ \pm}\right)^{-1} M_{e o} \\
0 & \left(M_{o o}^{ \pm}-M_{o e}\left(M_{e e}^{ \pm}\right)^{-1} M_{e o}\right)
\end{array}\right)^{-1}=\left(\begin{array}{cc}
1 & -\left(M_{e e}^{ \pm}\right)^{-1} M_{e o}\left(M_{o o}^{ \pm}-M_{o e}\left(M_{e e}^{ \pm}\right)^{-1} M_{e o}\right)^{-1} \\
0 & \left(M_{o o}^{ \pm}-M_{o e}\left(M_{e e}^{ \pm}\right)^{-1} M_{e o}\right)^{-1}
\end{array}\right) .
$$

The complete inversion is now performed in two separate steps: first compute for a given source field $\phi=\left(\phi_{e}, \phi_{0}\right)$ an intermediate result $\varphi=\left(\varphi_{e}, \varphi_{0}\right)$ by:

$$
\left(\begin{array}{c}
\varphi_{e} \\
\varphi_{o}
\end{array}\right)=\left(\begin{array}{ll}
M_{e e}^{ \pm} & 0 \\
M_{o e} & 1
\end{array}\right)^{-1}\left(\begin{array}{c}
\phi_{e} \\
\phi_{0}
\end{array}\right)=\left(\begin{array}{c}
\left(M_{e e}^{ \pm}\right)^{-1} \phi_{e} \\
-M_{o e}\left(M_{e e}^{ \pm}\right)^{-1} \phi_{e}+\phi_{0}
\end{array}\right)
$$

This step requires only the application of $M_{o e}$ and $\left(M_{e e}^{ \pm}\right)^{-1}$, the latter of which is given by Eq. (B.4). The final solution $\psi=\left(\psi_{e}, \psi_{0}\right)$ can then be computed with

$$
\left(\begin{array}{c}
\psi_{e} \\
\psi_{0}
\end{array}\right)=\left(\begin{array}{cc}
1 & \left(M_{e e}^{ \pm}\right)^{-1} M_{e o} \\
0 & \left(M_{o o}^{ \pm}-M_{o e}\left(M_{e e}^{ \pm}\right)^{-1} M_{e o}\right)
\end{array}\right)^{-1}\left(\begin{array}{c}
\varphi_{e} \\
\varphi_{o}
\end{array}\right)=\left(\begin{array}{c}
\varphi_{e}-\left(M_{e e}^{ \pm}\right)^{-1} M_{e o} \psi_{o} \\
\psi_{o}
\end{array}\right)
$$

where we defined

$$
\psi_{o}=\left(M_{o o}^{ \pm}-M_{o e}\left(M_{e e}^{ \pm}\right)^{-1} M_{e o}\right)^{-1} \varphi_{o}
$$

Therefore, the only inversion that has to be performed numerically is the one to generate $\psi_{0}$ from $\varphi_{0}$ and this inversion involves only an operator that is better conditioned than the original fermion operator.

Even/odd preconditioning can also be used for the mass non-degenerate Dirac operator $D_{h}$ Eq. (5). The corresponding equations follow immediately from the previous discussion and the definition from Eq. (B.14).

\section{Appendix C. Initialising the PHMC}

The function $1 / \sqrt{s}$ in the interval $[\epsilon, 1]$ can be approximated using polynomials or rational functions of different sorts. In the tmLQCD package we use Chebysheff polynomials, which are easy to construct. They can be constructed as to provide a desired overall precision in the interval $[\epsilon, 1]$.

As discussed in Section 2.3, the roots of the polynomial $P_{n, \epsilon}$ are needed for the evaluation of the force. Even though the roots come in complex conjugate pairs, for our case the roots cannot be computed analytically, hence we need to determine them numerically. Such an evaluation requires usually high precision. This is why these roots need to be determined before a PHMC run using an external program, i.e. they cannot be computed at the beginning of a run in the hmc_tm program. 
Such an external program ships with the tmLQCD code, which is located in the util/laguere directory. ${ }^{1}$ It is based on Laguerre's method and uses the Class Library for Numbers (CLN) [53], which provides arbitrary precision data types. In order to compute roots the CLN library must be available, which is free software.

Taking for granted that the CLN library is available, the procedure for computing the roots is as follows: assuming the non-degenerate Dirac operator has eigenvalues in the interval $\left[\tilde{s}_{\min }, \tilde{s}_{\max }\right]$, i.e. $\epsilon=\tilde{s}_{\min } / \tilde{s}_{\max }$, and the polynomial degree is $n$. Edit the file chebyRoot. H and set the variable EPSILON to the value of $\epsilon$. Moreover, set the variable MAXPOW to the degree $n$. Adapt the Makefile to your local installation and compile the code by typing make. After running the ChebyRoot program successfully, you should find two files in the directory

(1) Square_root_BR_roots.dat:

which contains the roots of the polynomial in bit-reverse order [23].

(2) normierungLocal. dat:

which contains a normalisation constant.

Copy these two files into the directory where you run the code and adjust the input parameters to match exactly the values used for the root computation. I.e. the input parameters StildeMin, StildeMax and DegreeofMDPolynomial must be set appropriately in the NDPOLY monomial.

The minimal and maximal eigenvalue of the non-degenerate flavour doublet can be computed as an online measurement. The frequency can be specified in the NDPOLY monomial with the input parameter ComputeEVFreq and they are written to the file called phme. data. Note that this is not a cheap operation in terms of computer time. However, if the approximation interval of the polynomial is chosen wrongly the algorithm performance might deteriorate drastically, in particular if the upper bound is set wrongly. It is therefore advisable to introduce some security measure in particular in the value of $\tilde{s}_{\max }$.

\section{References}

[1] A. Ukawa, Nucl. Phys. Proc. Suppl. 106 (2002) 195.

[2] C. Urbach, K. Jansen, A. Shindler, U. Wenger, Comput. Phys. Commun. 174 (2006) 87.

[3] M. Lüscher, Comput. Phys. Commun. 165 (2005) 199.

[4] M.A. Clark, A.D. Kennedy, Phys. Rev. Lett. 98 (2007) 051601

[5] M.A. Clark, A.D. Kennedy, Phys. Rev. D 75 (2007) 011502.

[6] A. Ali Khan, et al., Nucl. Phys. Proc. Suppl. 129 (2004) 853.

[7] A. Ali Khan, et al., Phys. Lett. B 564 (2003) 235.

[8] W. Kamleh, M.J. Peardon, PoS LAT2005 (2006) 106.

[9] M. Lüscher, JHEP 0712 (2007) 011.

[10] C.-N. Yang, R.L. Mills, Phys. Rev. 96 (1954) 191.

[11] K.G. Wilson, Phys. Rev. D 10 (1974) 2445.

[12] R. Frezzotti, P.A. Grassi, S. Sint, P. Weisz, JHEP 0108 (2001) 058.

[13] R. Frezzotti, G.C. Rossi, Nucl. Phys. Proc. Suppl. 128 (2004) 193.

[14] T. Chiarappa, et al., Eur. Phys. J. C 50 (2007) 373.

[15] S. Duane, A.D. Kennedy, B.J. Pendleton, D. Roweth, Phys. Lett. B 195 (1987) 216

[16] M. Hasenbusch, Phys. Lett. B 519 (2001) 177.

[17] M. Hasenbusch, K. Jansen, Nucl. Phys. B 659 (2003) 299

[18] M. Hasenbusch, Nucl. Phys. Proc. Suppl. 129 (2004) 27.

[19] M. Della Morte, et al., Comput. Phys. Commun. 156 (2003) 62.

[20] M.J. Peardon, J. Sexton, Nucl. Phys. Proc. Suppl. 119 (2003) 985.

[21] T. Takaishi, P. de Forcrand, Phys. Rev. E 73 (2006) 036706.

[22] J.C. Sexton, D.H. Weingarten, Nucl. Phys. B 380 (1992) 665.

[23] R. Frezzotti, K. Jansen, Phys. Lett. B 402 (1997) 328.

[24] R. Frezzotti, K. Jansen, Nucl. Phys. B 555 (1999) 395.

[25] R. Frezzotti, K. Jansen, Nucl. Phys. B 555 (1999) 432.

[26] M. Lüscher, Nucl. Phys. B 418 (1994) 637.

[27] B. Bunk, S. Elser, R. Frezzotti, K. Jansen, Comput. Phys. Commun. 118 (1999) 95.

[28] W. Press, S. Teukolsky, W. Vetterling, B. Flannery, Numerical Recipes in C, second ed., Cambridge University Press, Cambridge, UK, 1992.

[29] T.A. DeGrand, P. Rossi, Comput. Phys. Commun. 60 (1990) 211.

[30] K. Jansen, C. Liu, Comput. Phys. Commun. 99 (1997) 221.

[31] C.T. Sachrajda, G. Villadoro, Phys. Lett. B 609 (2005) 73.

[32] Y. Saad, Iterative Methods for Sparse Linear Systems, second ed., SIAM, 2003.

[33] P. Weisz, Nucl. Phys. B 212 (1983) 1.

[34] http://www-unix.mcs.anl.gov/mpi/.

[35] T. Chiarappa, et al., Comput. Sci. Disc. 01 (2008) 015001.

[36] M. Lüscher, Nucl. Phys. Proc. Suppl. 106 (2002) 21.

[37] M. Lüscher, http://luscher.web.cern.ch/luscher/QCDpm.

[38] R.G. Edwards, B. Joo, Nucl. Phys. Proc. Suppl. 140 (2005) 832.

[39] R.C. Brower, A.R. Levi, K. Orginos, Nucl. Phys. Proc. Suppl. 42 (1995) 855.

[40] P. Boucaud, et al., Comput. Phys. Commun. 179 (2008) 695.

[41] A. Meister, Numerik linearer Gleichungssysteme, Vieweg, 1999.

[42] T. Chiarappa, et al., Nucl. Phys. Proc. Suppl. 140 (2005) 853.

[43] G.L.G. Sleijpen, H.A.V. der Vorst, SIAM J. Matrix Anal. Appl. 17 (1996) 401.

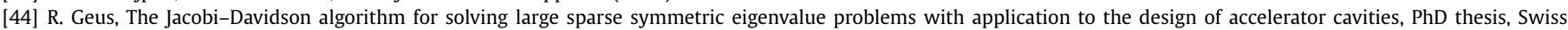
Federal Institute of Technology, Zürich, 2002.

[45] C. Morningstar, M.J. Peardon, Phys. Rev. D 69 (2004) 054501.

[46] M. Lüscher, Comput. Phys. Commun. 79 (1994) 100.

1 We thank Istvan Montvay for providing us with his code. 
[47] M. Lüscher, http://luscher.web.cern.ch/luscher/ranlux/.

[48] http://cssm.sasr.edu.au/ildg/.

[49] T. Yoshie, PoS LATTICE2008 (2008) 019.

[50] USQCD, http://usqcd.jlab.org/usqcd-docs/c-lime/.

[51] http://www.scidac.gov/.

[52] http://www.netlib.org/lapack/.

[53] http://www.ginac.de/CLN/. 